\title{
Improved double Fourier series on a sphere and its application to a semi-implicit semi-Lagrangian shallow water model
}

\author{
Hiromasa Yoshimura ${ }^{1}$ \\ ${ }^{1}$ Meteorological Research Institute, Japan Meteorological Agency, 1-1 Nagamine, Tsukuba, Ibaraki 305-0052, Japan \\ Correspondence to: Hiromasa Yoshimura (hyoshimu@mri-jma.go.jp)
}

\begin{abstract}
The computational cost of a spectral model using spherical harmonics (SH) increases significantly at high resolution because the transform method with $\mathrm{SH}$ requires $\mathrm{O}\left(N^{3}\right)$ operations, where $N$ is the truncation wavenumber. One way to solve this problem is to use double Fourier series (DFS) instead of $\mathrm{SH}$, which requires $\mathrm{O}\left(N^{2} \log N\right)$ operations. This paper proposes a new DFS method that improves the numerical stability of the model compared with the conventional DFS methods by

10 adopting the following two improvements: a new expansion method that employs the least-squares method (or the Galerkin method) to calculate the expansion coefficients in order to minimize the error caused by wavenumber truncation, and new basis functions that satisfy the continuity of both scalar and vector variables at the poles. In the semi-implicit semi-Lagrangian shallow water model using the new DFS method, the Williamson test cases 2 and 5 and the Galewsky test case give stable results without the appearance of high-wavenumber noise near the poles, even without using horizontal diffusion and a zonal Fourier filter. The new DFS model is faster than the SH model, especially at high resolutions, and gives almost the same results.
\end{abstract}

\section{Introduction}

Global spectral atmospheric models using the spectral transform method with spherical harmonics ( $\mathrm{SH}$ ) as basis functions are widely used. They are used in the Japan Meteorological Agency (JMA, 2019) and the Meteorological Research Institute (MRI; Yukimoto et al., 2011, 2019) for a range of applications, including operational weather prediction, operational seasonal prediction, and global warming projection. The spectral model has the advantage that the accuracy in horizontal derivatives is good, and the semi-implicit scheme, which improves numerical stability, can be easily applied because the Helmholtz equation and the Poisson equation are easily solved in spectral space. The application of the semi-implicit semi-Lagrangian scheme allows for timesteps longer than the Courant-Friedrichs-Lewy (CFL) condition, which makes the model computationally efficient. In the spectral model using $\mathrm{SH}$, the Legendre transform used in the latitudinal direction significantly increases the computational cost at high resolutions since the Legendre transform usually requires $\mathrm{O}\left(N^{3}\right)$ operations and $\mathrm{O}\left(N^{3}\right)$ memory usage, where $N$ is the truncation wavenumber. One way to reduce the operation count and the memory usage at high resolutions with large $N$ is to use the fast Legendre transform (Suda, 2005; Tygert, 2008; Wedi et al., 2013), which requires only $\mathrm{O}\left(N^{2}(\log N)^{3}\right)$ operations, although the accuracy is compromised to reduce the operation count. Dueben et al. (2020) presented global simulations of the atmosphere at $1.45 \mathrm{~km}$ grid-spacing in the $\mathrm{SH}$ model using the fast Legendre transform. 
Another approach used to improve the Legendre transform is on-the-fly computation of the associated Legendre functions (Schaeffer, 2013; Ishioka, 2018), which still requires $\mathrm{O}\left(N^{3}\right)$ operations but requires only $\mathrm{O}\left(N^{2}\right)$ memory usage. This small memory usage also contributes to speeding up calculations by taking advantage of the cache memory.

Another way to reduce the operation count and the memory usage in the global spectral model is to use double Fourier series 5 (DFS) as basis functions. In the DFS model, the fast Fourier transform (FFT; Cooley and Tukey, 1965; Swarztrauber, 1982) is used not only in the longitudinal (zonal) direction but also in the latitudinal (meridional) direction. The FFT requires only $\mathrm{O}\left(N^{2} \log N\right)$ operations and $\mathrm{O}(N)$ or $\mathrm{O}\left(N^{2}\right)$ memory usage, and it is much faster than the fast Legendre transform.

In DFS models (and also in SH models), the scalar variable $F(\lambda, \theta)$ is zonally expanded as

$$
F(\lambda, \theta) \cong \sum_{m=-M}^{M} F_{m}(\theta) e^{i m \lambda},
$$

10 where $\lambda$ is longitude, $\theta$ is colatitude, and $M$ is the zonal truncation wavenumber. Several methods have been proposed for meridional expansion with DFS. Merilees (1973b), Boer and Steinberg (1975), and Spotz et al. (1998) performed the Fourier transform meridionally along a great circle. Spotz et al. (1998) showed that by using the spherical harmonic filter, the explicit DFS shallow water model using the pseudo-spectral method can produce results comparable with the SH model in terms of accuracy and stability. However, the spherical harmonic filter consists of the forward SH transform (from grid space to spectral

15 space) followed by the inverse SH transform (from spectral space to grid space), which increases the computational cost.

Orszag (1974) and Boyd (1978) expanded $F_{m}(\theta)$ meridionally as

$$
\begin{gathered}
F_{m}(\theta) \cong \begin{cases}f_{m}(\theta) & \text { for even } m, \\
\sin \theta f_{m}(\theta) & \text { for odd } m,\end{cases} \\
f_{m}(\theta) \equiv \sum_{n=0}^{N} f_{n, m} \cos n \theta,
\end{gathered}
$$

where $N$ is the meridional truncation wavenumber. The coefficients $f_{n, m}$ for odd $m$ are calculated from the forward Fourier cosine transform of $F_{m}(\theta) / \sin \theta$. Orszag (1974) imposed the following conditions at the poles:

$$
f_{m}(0)=0 \text { and } f_{m}(\pi)=0 \text { for }|m| \geq 2,
$$

which can be expressed in terms of the expansion coefficients $f_{n, m}$ as

$$
\sum_{\substack{n=0 \\ n \text { is even }}}^{N} f_{n, m}=0 \text { and } \sum_{\substack{n=1 \\ n \text { is odd }}}^{N} f_{n, m}=0 \text { for }|m| \geq 2 .
$$

Satisfying the above conditions ensures that the scalar variable $F(\lambda, \theta)$ and its gradient $\nabla F$ are continuous at the poles. In Orszag (1974), only $f_{N-1, m}$ and $f_{N, m}$ were modified to satisfy Eq. (4), but this is not the best way to satisfy the same conditions as Eq. (3) or Eq. (4), as will be shown in Sect. 3.

Yee (1981) and Layton and Spotz (2003) expanded $F_{m}(\theta)$ as 


$$
F_{m}(\theta)= \begin{cases}\sum_{n=0}^{N} F_{n, m} \cos n \theta & \text { for even } m, \\ \sum_{n=1}^{N} F_{n, m} \sin n \theta & \text { for odd } m .\end{cases}
$$

In the semi-implicit semi-Lagrangian shallow water model in Layton and Spotz (2003), the spherical harmonic filter was applied to the prognostic variables for stability and accuracy. Layton and Spotz (2003) explained that the expansion with Eq. (5) permits discontinuity at the poles and nonisotropic waves, which may lead to a prohibitive timestep restriction and 5 numerical instability, and these problems can be avoided by applying the spherical harmonic filter.

Cheong (2000a, 2000b) proposed expanding $F_{m}(\theta)$ as

$$
F_{m}(\theta) \cong \begin{cases}\sum_{n=0}^{N} F_{n, m} \cos n \theta & \text { for } m=0, \\ \sum_{n=1}^{N} F_{n, m} \sin n \theta & \text { for odd } m, \\ \sum_{n=1}^{N} F_{n, m}^{\prime} \sin \theta \sin n \theta & \text { for even } m(\neq 0) .\end{cases}
$$

The meridional basis functions $\sin \theta \sin n \theta$ for even $m(\neq 0)$ are different from Eq. (5). The coefficients $F_{n, m}^{\prime}$ for even $m(\neq$ 0 ) are calculated by forward Fourier sine transform of $F_{m}(\theta) / \sin \theta$. The basis functions in Eq. (6) automatically satisfy the 10 same conditions at the poles as Eq. (3) for even $m$, and guarantee the continuity of the scalar variable $F$ at the poles, which is an advantage compared with the basis functions in Eq. (5). However, Eq. (6) does not automatically satisfy the conditions in Eq. (3) for odd $m$, and does not guarantee the continuity of $\nabla F$ at the poles. The shallow water model and the vorticity equation model using a semi-implicit Eulerian scheme ran stably using high-order horizontal diffusion with $\mathrm{O}\left(N^{2}\right)$ operations to smooth out the high-wavenumber components (Cheong, 2000b; Cheong et al., 2002; Kwon et al., 2004). The semi-implicit Eulerian

15 hydrostatic atmospheric model also ran stably with high-order horizontal diffusion (Cheong, 2006; Koo and Hong, 2013; Park et al., 2013). However, the computational results of these models appear to be a little different from (slightly worse than) the models using SH. One reason for this seems to be the appearance of high-wavenumber oscillation resulting from the meridional wavenumber truncation with $N \cong 2 J / 3$ or $J / 2$ for even $m(\neq 0)$ (See Sect. 3), and the use of strong high-order horizontal diffusion to smooth out the oscillation, where $J$ is the number of grid points in the latitudinal direction.

20 Yoshimura and Matsumura (2005) and Yoshimura (2012) stably ran the two-time-level semi-implicit semi-Lagrangian hydrostatic and nonhydrostatic atmospheric models using the DFS basis functions of Cheong in Eq. (6). These models used meridional truncation with $N \cong J$, and $U=u \sin \theta$ and $V=v \sin \theta$ (instead of $u / \sin \theta$ and $v / \sin \theta$ ) were transformed from grid space to spectral space, where $u$ is the zonal wind and $v$ is the meridional wind. These models used the same horizontal diffusion as the SH models, and did not require the strong high-order horizontal diffusion. The results of these models were very similar to those of the SH models. However, we found the following two problems in these models: 
1. High wavenumber noise appears near the poles.

2. The meridional wavenumber truncation $N$ needs to be equal to $J$ for even $m(\neq 0)$ because $N<J$ (e.g., $N \cong 2 J / 3)$ causes the high-wavenumber oscillation and the numerical instability (See Sect. 3).

To solve these problems, we propose a new DFS method that adopts the following two improvements:

5 1. A new expansion method to calculate DFS expansion coefficients of scalar and vector variables, which adopts the leastsquares method (or the Galerkin method) to minimize the error due to the meridional wavenumber truncation.

2 New DFS basis functions that automatically satisfy the pole conditions in Eq. (3), which guarantee continuity of not only scalar variables but also vector variables at the poles.

We also use the Galerkin method to solve partial differential equations such as the Poisson equation and the shallow water 10 equations.

Section 2 describes the details of the new DFS method using the new DFS expansion method and the new DFS basis functions. Section 3 examines the error due to the wavenumber truncation in the new DFS method, Orszag's DFS method, and Cheong's DFS method. Section 4 describes how to integrate the semi-implicit semi-Lagrangian shallow water model using the new DFS method. Section 5 compares the results of the model using the new DFS method with those using the old DFS method of Yoshimura and Matsumura (2005), and with those using the SH method. Section 6 presents conclusions and perspectives.

\section{Improved double Fourier series on the sphere}

\subsection{New basis functions for a scalar variable}

We propose the following new DFS basis functions that automatically satisfy the continuity conditions at the poles in Eq. (3). The scalar variable $T(\lambda, \theta)$ is expanded zonally as

$$
T(\lambda, \theta) \cong \sum_{m=0}^{M} T_{m}^{\mathrm{c}}(\theta) \cos m \lambda+\sum_{m=1}^{M} T_{m}^{\mathrm{s}}(\theta) \sin m \lambda,
$$

and the variables $T_{m}^{\mathrm{c}}(\theta)$ and $T_{m}^{\mathrm{s}}(\theta)$ are meridionally expanded as

$$
T_{m}^{\mathrm{c}}(\theta) \cong T_{m}^{\mathrm{c}, N}(\theta) \equiv \begin{cases}\sum_{n=0}^{N} T_{n, m}^{\mathrm{c}} \cos n \theta & \text { for } m=0, \\ \sum_{n=0}^{N-1} T_{n, m}^{\mathrm{c}} \sin \theta \cos n \theta & \text { for } m=1, \\ \sum_{n=1}^{N-1} T_{n, m}^{\mathrm{c}} \sin \theta \sin n \theta & \text { for even } m \geq 2, \\ \sum_{n=1}^{N-2} T_{n, m}^{\mathrm{c}} \sin ^{2} \theta \sin n \theta & \text { for odd } m \geq 3,\end{cases}
$$




$$
T_{m}^{S}(\theta) \cong T_{m}^{\mathrm{s}, N}(\theta) \equiv \begin{cases}\sum_{n=0}^{N-1} T_{n, m}^{\mathrm{s}} \sin \theta \cos n \theta & \text { for } m=1, \\ \sum_{n=1}^{N-1} T_{n, m}^{\mathrm{s}} \sin \theta \sin n \theta & \text { for even } m \geq 2, \\ \sum_{n=1}^{N-2} T_{n, m}^{\mathrm{s}} \sin ^{2} \theta \sin n \theta & \text { for odd } m \geq 3 .\end{cases}
$$

In Eq. (7), $\cos m \lambda$ and $\sin m \lambda$ are used instead of $e^{i m \lambda}$ as zonal basis functions for convenience in calculating the expansion coefficients using the least-squares method described later in Sects. 2.3 and 2.7. In Eq. (8), the meridional basis functions $\sin ^{2} \theta \sin n \theta$ for odd $m \geq 3$ are especially different from Cheong's basis functions in Eq. (6). Either $\sin n \theta \operatorname{or} \sin \theta \cos n \theta$

5 can be used as the basis functions for $m=1$ because it can be shown using Eq. (A2) from Appendix A that $\sin \theta \cos n \theta(n=0, \ldots, N-1)$ are the linear combination of $\sin n \theta(n=1, \ldots, N)$, and vice versa. Here we use $\sin \theta \cos n \theta$ for $m=1$ because it can be more easily divided by $\sin \theta$, which is convenient for calculating $\nabla T$.

Using Eq. (A2), Eq. (8) can be transformed as follows:

$$
T_{m}^{c, N}(\theta)= \begin{cases}\sum_{n=0}^{N} T_{n, m}^{c^{\prime}} \cos n \theta & \text { for even } m, \\ \sum_{n=1}^{N} T_{n, m}^{c^{\prime}} \sin n \theta & \text { for odd } m,\end{cases}
$$

10 where

$$
\begin{array}{rlrl}
T_{n, m}^{\mathrm{c}^{\prime}}= & T_{n, m}^{\mathrm{c}} & \text { for } m=0, \\
T_{n, m}^{\mathrm{c}^{\prime}}= & \frac{T_{n-1, m}^{\mathrm{c}}-T_{n+1, m}^{\mathrm{c}}}{2} & \text { for } m=1 \\
& \text { except for } T_{1, m}^{\mathrm{c}^{\prime}}=\frac{2 T_{0, m}^{\mathrm{c}}-T_{2, m}^{\mathrm{c}}}{2}, & \\
T_{n, m}^{\mathrm{c}^{\prime}}=\frac{-T_{n-1, m}^{\mathrm{c}}+T_{n+1, m}^{\mathrm{c}}}{2} & \text { for even } m \geq 2, \\
T_{n, m}^{\mathrm{c}^{\prime}}=\frac{-T_{n-2, m}^{\mathrm{c}}+2 T_{n, m}^{\mathrm{c}}-T_{n+2, m}^{\mathrm{c}}}{4} & \text { for odd } m \geq 3 \\
& \text { except for } T_{1, m}^{\mathrm{c}^{\prime}}=\frac{3 T_{1, m}^{\mathrm{c}}-T_{3, m}^{\mathrm{c}}}{4} . &
\end{array}
$$

The equations for $T_{m}^{s, N}(\theta)$ and $T_{n, m}^{\mathrm{s}^{\prime}}$ for $m \geq 1$ are the same as Eqs. (9) and (10), except that the superscript $\mathrm{c}$ is replaced with the superscript s. The upper limit of $n$ for each $m$ in Eq. (8) is determined so that the upper limit of $n$ for each $m$ in Eq. (9) becomes $N$.

When calculating the values of $T_{m}^{\mathrm{c}, N}(\theta)\left(T_{m}^{\mathrm{s}, N}(\theta)\right)$ in grid space from $T_{n, m}^{\mathrm{c}}\left(T_{n, m}^{\mathrm{s}}\right)$ in spectral space, the coefficients $T_{n, m}^{\mathrm{c}^{\prime}}$ $\left(T_{n, m}^{\mathrm{s}^{\prime}}\right)$ are calculated from $T_{n, m}^{\mathrm{c}}\left(T_{n, m}^{\mathrm{s}}\right)$ using Eq. (10) and inverse discrete cosine and sine transforms are performed using Eq. 
(9) (See Sect. 2.10). The calculation of $T_{n, m}^{\mathrm{c}}\left(T_{n, m}^{\mathrm{s}}\right)$ in spectral space from $T_{m}^{\mathrm{c}}(\theta)\left(T_{m}^{\mathrm{s}}(\theta)\right)$ in grid space is described in Sect. 2.3 below.

The truncated variable $T^{N, M}(\lambda, \theta)$ is defined as

$$
T^{N, M}(\lambda, \theta) \equiv \sum_{m=0}^{M} T_{m}^{\mathrm{c}, N}(\theta) \cos m \lambda+\sum_{m=1}^{M} T_{m}^{s, N}(\theta) \sin m \lambda .
$$

5 From Eq. (8), the values of $T_{m}^{\mathrm{c}, N}(\theta)$ at the poles are finite for $m=0$, and the values of $T_{m}^{\mathrm{c}, N}(\theta)$ and $T_{m}^{\mathrm{s}, N}(\theta)$ at the poles are zero for $m \neq 0$. Therefore $T^{N, M}(\lambda, \theta)$ is continuous at the poles.

\subsection{Gradient of a scalar variable}

The gradient $\nabla T^{N, M}=\left(T_{\lambda}^{N, M}, T_{\phi}^{N, M}\right)$ is obtained as follows:

$$
\begin{aligned}
& T_{\lambda}^{N, M} \equiv \frac{1}{a \sin \theta} \frac{\partial T^{N, M}}{\partial \lambda}=\sum_{m=1}^{M} T_{\lambda, m}^{\mathrm{c}, N}(\theta) \cos m \lambda+\sum_{m=1}^{M} T_{\lambda, m}^{\mathrm{s}, N}(\theta) \sin m \lambda, \\
& T_{\lambda, m}^{\mathrm{c}, N}(\theta) \equiv \frac{m}{a \sin \theta} T_{m}^{\mathrm{s}, N}(\theta), \quad T_{\lambda, m}^{\mathrm{s}, N}(\theta) \equiv-\frac{m}{a \sin \theta} T_{m}^{\mathrm{c}, N}(\theta), \\
& T_{\phi}^{N, M} \equiv \frac{1}{a} \frac{\partial T^{N, M}}{\partial \phi}=-\frac{1}{a} \frac{\partial T^{N, M}}{\partial \theta}=\sum_{m=0}^{M} T_{\phi, m}^{\mathrm{c}, N}(\theta) \cos m \lambda+\sum_{m=1}^{M} T_{\phi, m}^{\mathrm{s}, N}(\theta) \sin m \lambda, \\
& T_{\phi, m}^{\mathrm{c}, N}(\theta) \equiv-\frac{1}{a} \frac{\partial T_{m}^{c, N}(\theta)}{\partial \theta}, \quad T_{\phi, m}^{\mathrm{s}, N}(\theta) \equiv-\frac{1}{a} \frac{\partial T_{m}^{\mathrm{s}, N}(\theta)}{\partial \theta},
\end{aligned}
$$

where $a$ is the radius of the earth, and $\phi$ is the latitude. From Eqs. (12b), (8) and (A2) we obtain

$$
T_{\lambda, m}^{\mathrm{c}, N}(\theta)=\left\{\begin{array}{lr}
0 & \text { for } m=0, \\
\sum_{n=1}^{N} T_{\lambda, n, m}^{\mathrm{c}} \cos n \theta & \text { for } m=1, \\
\sum_{n=1}^{N} T_{\lambda, n, m}^{\mathrm{c}} \sin n \theta & \text { for even } m \geq 2, \\
\sum_{n=0}^{N} T_{\lambda, n, m}^{\mathrm{c}} \cos n \theta\left(=\sum_{n=1}^{N-1} T_{\lambda, n, m}^{\mathrm{c}^{\prime}} \sin \theta \sin n \theta\right) & \text { for odd } m \geq 3,
\end{array}\right.
$$

15 where

$$
\begin{array}{rlrl}
T_{\lambda, n, m}^{\mathrm{c}} & =\frac{1}{a} T_{n, m}^{\mathrm{s}} & \text { for } m=1, \\
T_{\lambda, n, m}^{\mathrm{c}}=\frac{1}{a} m T_{n, m}^{\mathrm{s}} & \text { for even } m \geq 2, \\
T_{\lambda, n, m}^{\mathrm{c}}=\frac{1}{a} \frac{m\left(-T_{n-1, m}^{\mathrm{s}}+T_{n+1, m}^{\mathrm{s}}\right)}{2} & \text { for odd } m \geq 3 .
\end{array}
$$


The equation for $T_{\lambda, m}^{\mathrm{s}, N}(\theta)$ is the same as Eq. (14), except that the subscript $\mathrm{c}$ is replaced with s. The equations for $T_{\lambda, n, m}^{\mathrm{s}}$ are the same as Eq. (15), except that $T_{\lambda, n, m}^{\mathrm{c}}$ and $T_{n, m}^{\mathrm{s}}$ are replaced with $T_{\lambda, n, m}^{\mathrm{s}}$ and $-T_{n, m}^{\mathrm{c}}$, respectively. From Eqs. (13b), (9), and (10) we obtain

$$
T_{\phi, m}^{c, N}(\theta)=\left\{\begin{array}{lr}
\sum_{n=1}^{N} T_{\phi, n, m}^{c} \sin n \theta & \text { for } m=0, \\
\sum_{n=1}^{N} T_{\phi, n, m}^{\mathrm{c}} \cos n \theta & \text { for } m=1, \\
\sum_{n=1}^{N} T_{\phi, n, m}^{\mathrm{c}} \sin n \theta & \text { for even } m \geq 2, \\
\sum_{n=0}^{N} T_{\phi, n, m}^{c} \cos n \theta\left(=\sum_{n=1}^{N-1} T_{\phi, n, m}^{c^{\prime}} \sin \theta \sin n \theta\right) & \text { for odd } m \geq 3,
\end{array}\right.
$$

5 where

$$
\begin{aligned}
& T_{\phi, n, m}^{\mathrm{c}}=-\frac{1}{a}\left(-n T_{n, m}^{\mathrm{c}}\right) \quad \text { for } m=0, \\
& T_{\phi, n, m}^{\mathrm{c}}=-\frac{1}{a}\left[\frac{n\left(T_{n-1, m}^{\mathrm{c}}-T_{n+1, m}^{\mathrm{c}}\right)}{2}\right] \quad \text { for } m=1 \\
& \text { except for } T_{\phi, 1, m}^{\mathrm{c}}=-\frac{1}{a}\left[\frac{2 T_{0, m}^{\mathrm{c}}-T_{2, m}^{\mathrm{c}}}{2}\right] \text {, } \\
& T_{\phi, n, m}^{c}=-\frac{1}{a}\left[\frac{n\left(T_{n-1, m}^{c}-T_{n+1, m}^{c}\right)}{2}\right] \quad \text { for even } m \geq 2 \text {, } \\
& T_{\phi, n, m}^{\mathrm{c}}=-\frac{1}{a}\left[\frac{n\left(-T_{n-2, m}^{\mathrm{c}}+2 T_{n, m}^{\mathrm{c}}-T_{n+2, m}^{\mathrm{c}}\right)}{4}\right] \quad \text { for odd } m \geq 3 \\
& \text { except for } T_{\theta, 1, m}^{\mathrm{c}}=-\frac{1}{a}\left[\frac{\left(3 T_{1, m}^{\mathrm{c}}-T_{3, m}^{\mathrm{c}}\right)}{4}\right] \text {. }
\end{aligned}
$$

The equations for $T_{\phi, m}^{\mathrm{s} N}(\theta)$ and $T_{\phi, n, m}^{\mathrm{s}}$ for $m \geq 1$ are the same as Eqs. (16) and (17), except that the subscript $\mathrm{c}$ is replaced with s. From Eqs. (14) to (17), it can be seen that $T_{\lambda, m}^{\mathrm{c}, N}(\theta), T_{\lambda, m}^{\mathrm{s}, N}(\theta), T_{\phi, m}^{\mathrm{c}, N}(\theta)$, and $T_{\phi, m}^{\mathrm{s}, N}(\theta)$ at the poles are finite for $m=1$ and zero for $m \neq 1$, and moreover the following relations are satisfied for $m=1$ :

$$
\begin{aligned}
& T_{\lambda, m=1}^{\mathrm{c}, N}(\theta)=-T_{\phi, m=1}^{\mathrm{s}, N}(\theta)\left(=\frac{1}{a} \sum_{n=1}^{N-1} T_{n, m=1}^{\mathrm{s}}\right) \quad \text { at } \theta=0 \text { (North Pole), } \\
& T_{\lambda, m=1}^{\mathrm{s}, N}(\theta)=T_{\phi, m=1}^{\mathrm{c}, N}(\theta)\left(=-\frac{1}{a} \sum_{n=1}^{N-1} T_{n, m=1}^{\mathrm{c}}\right) \quad \text { at } \theta=0 \text { (North Pole), } \\
& T_{\lambda, m=1}^{\mathrm{c}, N}(\theta)=T_{\phi, m=1}^{\mathrm{s}, N}(\theta)\left(=\frac{1}{a} \sum_{n=1}^{N-1}(-1)^{n} T_{n, m=1}^{\mathrm{s}}\right) \quad \text { at } \theta=\pi \text { (South Pole), }
\end{aligned}
$$




$$
T_{\lambda, m=1}^{\mathrm{s}, N}(\theta)=-T_{\phi, m=1}^{\mathrm{c}, N}(\theta)\left(=-\frac{1}{a} \sum_{n=1}^{N-1}(-1)^{n} T_{n, m=1}^{\mathrm{c}}\right) \text { at } \theta=\pi \text { (South Pole). }
$$

Thus, it is guaranteed that $\nabla T^{N, M}=\left(T_{\lambda}^{N, M}, T_{\phi}^{N, M}\right)$ is continuous at the poles.

\subsection{New method to calculate expansion coefficients for a scalar variable}

One way to calculate the coefficients $T_{n, m}^{\mathrm{c}}\left(T_{n, m}^{\mathrm{s}}\right)$ from $T_{m}^{\mathrm{c}}(\theta)\left(T_{m}^{\mathrm{s}}(\theta)\right)$ in Eq. (8) is to perform a forward cosine transform 5 of $T_{m}^{\mathrm{c}}(\theta) / \sin \theta\left(T_{m}^{\mathrm{s}}(\theta) / \sin \theta\right)$ for $m=1$, a cosine transform of $T_{m}^{\mathrm{c}}(\theta) / \sin \theta\left(T_{m}^{\mathrm{s}}(\theta) / \sin \theta\right)$ for even $m \geq 2$, and a sine transform of $T_{m}^{\mathrm{c}}(\theta) / \sin ^{2} \theta\left(T_{m}^{\mathrm{s}}(\theta) / \sin ^{2} \theta\right)$ for odd $m \geq 3$. However, this approach with the meridional wavenumber truncation $N<J$ leads to the large high-wavenumber oscillation as in Cheong's basis functions for even $m \geq 2$ (See Sect. 3). Dividing $T_{m}^{\mathrm{c}}(\theta)$ by $\sin ^{2} \theta$ reduces the numerical stability of the model more significantly than dividing $T_{m}^{\mathrm{c}}(\theta)$ by $\sin \theta$.

Here we propose a new method to calculate expansion coefficients using the least-squares method to minimize the error due

to the meridional wavenumber truncation. This method also avoids dividing $T_{m}^{\mathrm{c}}(\theta)$ by $\sin \theta$ or $\sin ^{2} \theta$ before the forward cosine or sine transforms. The coefficients $T_{n, m}^{\mathrm{c}}$ and $T_{n, m}^{\mathrm{s}}$ in Eq. (8) are calculated as follows. First, $T_{m}^{\mathrm{c}}(\theta)$ and $T_{m}^{\mathrm{s}}(\theta)$ in Eq. (8) are expanded like Eq. (5) as

$$
T_{m}^{\mathrm{c}}(\theta) \cong \tilde{T}_{m}^{\mathrm{c}, J}(\theta) \equiv \begin{cases}\sum_{n=0}^{J-1} \tilde{T}_{n, m}^{\mathrm{c}} \cos n \theta & \text { for even } m, \\ \sum_{n=1}^{J} \tilde{T}_{n, m}^{\mathrm{c}} \sin n \theta & \text { for odd } m,\end{cases}
$$

where $J$ is the number of meridional grid points, and the expansion coefficients $\widetilde{T}_{n, m}^{c}$ are calculated by the forward discrete cosine transform for even $m$ and the forward discrete sine transform for odd $m$ from the values of $T_{m}^{\mathrm{c}}(\theta)$ at the grid points (See Sect. 2.10). The equation for $T_{m}^{\mathrm{s}}(\theta)$ is the same as Eq. (19), except that the subscript $\mathrm{c}$ is replaced with $\mathrm{s}$.

Next, $T_{n, m}^{\mathrm{c}}$ and $T_{n, m}^{\mathrm{s}}$ are calculated using the least-squares method to minimize the following error $E$ (the squared $\mathrm{L}_{2}$ norm o $f$ the residual):

$$
E \equiv \frac{1}{2 \pi^{2}} \int_{0}^{2 \pi} \int_{0}^{\pi} R(\lambda, \theta)^{2} d \theta d \lambda,
$$

where the residual $R(\lambda, \theta)$ is

$$
R(\lambda, \theta) \equiv\left(\sum_{m=0}^{M} T_{m}^{\mathrm{c}, N}(\theta) \cos m \lambda+\sum_{m=1}^{M} T_{m}^{\mathrm{s}, N}(\theta) \sin m \lambda\right)-\left(\sum_{m=0}^{M} \tilde{T}_{m}^{\mathrm{c}, J}(\theta) \cos m \lambda+\sum_{m=1}^{M} \tilde{T}_{m}^{\mathrm{s}, J}(\theta) \sin m \lambda\right)
$$

From Eqs. (20), (21), and (A3), we derive

$$
E=\frac{1}{\pi} \int_{0}^{\pi}\left[\left(T_{m=0}^{\mathrm{c}, N}(\theta)-\tilde{T}_{m=0}^{\mathrm{c}, J}(\theta)\right)^{2}+\frac{1}{2} \sum_{m=1}^{M}\left(T_{m}^{\mathrm{c}, N}(\theta)-\widetilde{T}_{m}^{\mathrm{c}, J}(\theta)\right)^{2}+\frac{1}{2} \sum_{m=1}^{M}\left(T_{m}^{\mathrm{s}, N}(\theta)-\tilde{T}_{m}^{\mathrm{s}, J}(\theta)\right)^{2}\right] d \theta .
$$


Eqs. (9), (10), and (19) are substituted into Eq. (22). The equations $\partial E / \partial T_{n, m}^{c}=0$ and $\partial E / \partial T_{n, m}^{s}=0$ are used to calculate $T_{n, m}^{\mathrm{c}}$ and $T_{n, m}^{\mathrm{s}}$, respectively, so that $E$ is minimized.

From $\partial E / \partial T_{n, m}^{c}=0$ and Eq. (A4), we derive

$$
\begin{array}{ll}
\frac{\partial}{\partial T_{n, m}^{\mathrm{c}}}\left[2\left(T_{0, m}^{\mathrm{c}^{\prime}}-\tilde{T}_{0, m}^{\mathrm{c}}\right)^{2}+\sum_{n=1}^{N}\left(T_{n, m}^{\mathrm{c}^{\prime}}-\tilde{T}_{n, m}^{\mathrm{c}}\right)^{2}\right]=0 & \text { for even } m, \\
\frac{\partial}{\partial T_{n, m}^{\mathrm{c}}}\left[\sum_{n=1}^{N}\left(T_{n,}^{\mathrm{c}^{\prime}}-\tilde{T}_{n, m}^{\mathrm{c}}\right)^{2}\right]=0 & \text { for odd } m .
\end{array}
$$

From Eq. (23) and (10), we derive the following equations for $T_{n, m}^{\mathrm{c}}$.

For $m=0$,

$$
T_{n, m}^{\mathrm{c}}=\tilde{T}_{n, m}^{\mathrm{c}} \quad(0 \leq n \leq N) .
$$

For $m=1$,

$$
-T_{n-2, m}^{\mathrm{c}}+2 T_{n, m}^{\mathrm{c}}-T_{n+2, m}^{\mathrm{c}}=-2 \tilde{T}_{n-1, m}^{\mathrm{c}}+2 \tilde{T}_{n+1, m}^{\mathrm{c}} \quad(0 \leq n \leq N-1),
$$

with the exception of the following underlined values:

$$
\begin{array}{cc}
\underline{1} T_{1, m}^{c}-T_{3, m}^{c}=2 \tilde{T}_{2, m}^{c} & (n=1), \\
-\underline{2} T_{0, m}^{c}+2 T_{2, m}^{c}-T_{4, m}^{c}=-2 \tilde{T}_{1, m}^{c}+2 \tilde{T}_{3, m}^{c} & (n=2) .
\end{array}
$$

For even $m(\geq 2)$,

$$
-T_{n-2, m}^{\mathrm{c}}+2 T_{n, m}^{\mathrm{c}}-T_{n+2, m}^{\mathrm{c}}=2 \tilde{T}_{n-1, m}^{\mathrm{c}}-2 \tilde{T}_{n+1, m}^{\mathrm{c}} \quad(1 \leq n \leq N-1),
$$

with the exception of the following underlined values:

$$
\underline{3} T_{1, m}^{\mathrm{c}}-T_{3, m}^{\mathrm{c}}=\underline{4} \tilde{T}_{0, m}^{\mathrm{c}}-2 \tilde{T}_{2, m}^{\mathrm{c}} \quad(n=1) .
$$

For odd $m(\geq 3)$

$$
T_{n-4, m}^{\mathrm{c}}-4 T_{n-2, m}^{\mathrm{c}}+6 T_{n, m}^{\mathrm{c}}-4 T_{n+2, m}^{\mathrm{c}}+T_{n+4, m}^{\mathrm{c}}=-4 \tilde{T}_{n-2, m}^{\mathrm{c}}+8 \tilde{T}_{n, m}^{\mathrm{c}}-4 \tilde{T}_{n+2, m}^{\mathrm{c}}(1 \leq n \leq N-2),
$$

with the exception of the following underlined values:

$$
\begin{array}{cc}
\underline{10} T_{1, m}^{\mathrm{c}}-\underline{5} T_{3, m}^{\mathrm{c}}+T_{5, m}^{\mathrm{c}}=\underline{12} \tilde{T}_{1, m}^{\mathrm{c}}-4 \widetilde{T}_{3, m}^{\mathrm{c}} & (n=1), \\
\underline{5} T_{2, m}^{\mathrm{c}}-4 T_{4, m}^{\mathrm{c}}+T_{6, m}^{\mathrm{c}}=8 \tilde{T}_{2, m}^{\mathrm{c}}-4 \widetilde{T}_{4, m}^{\mathrm{c}} & (n=2), \\
-\underline{5} T_{1, m}^{\mathrm{c}}+6 T_{3, m}^{\mathrm{c}}-4 T_{5, m}^{\mathrm{c}}+T_{7, m}^{\mathrm{c}}=-4 \tilde{T}_{1, m}^{\mathrm{c}}+8 \tilde{T}_{3, m}^{\mathrm{c}}-4 \tilde{T}_{5, m}^{\mathrm{c}} & (n=3) .
\end{array}
$$

From Eq. (24d), two linear simultaneous equations with penta-diagonal matrices,

$$
\left[\begin{array}{cccccccc}
* & * & * & 0 & 0 & 0 & \cdots & 0 \\
* & * & * & * & 0 & 0 & \cdots & 0 \\
* & * & * & * & * & 0 & \cdots & 0 \\
0 & * & * & * & * & * & \cdots & 0 \\
0 & 0 & 0 & \cdots & 0 & * & * & *
\end{array}\right]\left[\begin{array}{c}
T_{0, m}^{\mathrm{c}} \\
T_{2, m}^{\mathrm{c}} \\
T_{4, m}^{\mathrm{c}} \\
T_{6, m}^{\mathrm{c}} \\
\vdots \\
\vdots
\end{array}\right]=\left[\begin{array}{c}
* \\
* \\
* \\
* \\
\vdots \\
*
\end{array}\right],\left[\begin{array}{cccccccc}
* & * & * & 0 & 0 & 0 & \cdots & 0 \\
* & * & * & * & 0 & 0 & \cdots & 0 \\
* & * & * & * & * & 0 & \cdots & 0 \\
0 & * & * & * & * & * & \cdots & 0 \\
& & & & \vdots & & & \\
0 & 0 & 0 & \cdots & 0 & * & * & *
\end{array}\right]\left[\begin{array}{c}
T_{1, m}^{\mathrm{c}} \\
T_{3, m}^{\mathrm{c}} \\
T_{5, m}^{\mathrm{c}} \\
T_{7, m}^{\mathrm{c}} \\
\vdots \\
\vdots
\end{array}\right]=\left[\begin{array}{c}
* \\
* \\
* \\
* \\
\vdots \\
*
\end{array}\right]
$$

are derived. A penta-diagonal matrix can be LU decomposed as 


$$
\left[\begin{array}{cccccccc}
* & * & * & 0 & 0 & 0 & \cdots & 0 \\
* & * & * & * & 0 & 0 & \cdots & 0 \\
* & * & * & * & * & 0 & \cdots & 0 \\
0 & * & * & * & * & * & \cdots & 0 \\
0 & 0 & \cdots & 0 & * & * & * & * \\
0 & 0 & \cdots & 0 & 0 & * & * & *
\end{array}\right]=\mathbf{L} \mathbf{U}, \quad \mathbf{L} \equiv\left[\begin{array}{ccccccccc}
* & 0 & 0 & 0 & 0 & 0 & \cdots & 0 \\
* & * & 0 & 0 & 0 & 0 & \cdots & 0 \\
* & * & * & 0 & 0 & 0 & \cdots & 0 \\
0 & * & * & * & 0 & 0 & \cdots & 0 \\
& & & & \vdots & & & \\
0 & 0 & \cdots & 0 & * & * & * & 0 \\
0 & 0 & \cdots & 0 & 0 & * & * & *
\end{array}\right], \quad \mathbf{U} \equiv\left[\begin{array}{ccccccccc}
1 & * & * & 0 & 0 & 0 & \cdots & 0 \\
0 & 1 & * & * & 0 & 0 & \cdots & 0 \\
0 & 0 & 1 & * & * & 0 & \cdots & 0 \\
0 & 0 & 0 & 1 & * & * & \cdots & 0 \\
& & & & \vdots & & & \\
0 & 0 & \cdots & 0 & 0 & 0 & 1 & * \\
0 & 0 & \cdots & 0 & 0 & 0 & 0 & 1
\end{array}\right] .
$$

To solve $\mathbf{L U} \boldsymbol{x}=\boldsymbol{b}$, we solve $\mathbf{L} \boldsymbol{y}=\boldsymbol{b}$ with forward substitution first, and then solve $\mathbf{U} \boldsymbol{x}=\boldsymbol{y}$ with backward substitution. There are also other methods to solve Eq. (25). For example, the method using LU decomposition considering penta-diagonal matrices as $2 \times 2$ block tri-diagonal matrices makes SIMD operations more effective. The method using cyclic reduction for

5 block tri-diagonal matrices (e.g., Gander and Golub, 1997) is suitable for vectorization and parallelization. The calculation with these methods for each $m$ requires $\mathrm{O}(N)$ operations. The simultaneous equations with tri-diagonal matrices derived from Eqs. (24b, c) can be solved in a similar way to Eq. (25). Therefore, the calculation of $T_{n, m}^{\mathrm{c}}$ for all $m$ and $n$ with Eq. (24) requires only $\mathrm{O}\left(N^{2}\right)$ operations.

The equations for $T_{n, m}^{\mathrm{s}}$ are derived from $\partial E / \partial T_{n, m}^{\mathrm{s}}=0$; these are the same equations as Eqs. $(24 \mathrm{~b}, \mathrm{c}, \mathrm{d})$, except that the subscript $\mathrm{c}$ is replaced with $\mathrm{s}$.

\subsection{Relation between the least-squares method and Galerkin method for a scalar variable}

Here we discuss the relation between the least-squares method described above and the Galerkin method when calculating the expansion coefficients of a scalar variable.

From Eqs. (20) and (21) and the equations $\partial E / \partial T_{n, m}^{\mathrm{c}}=0$ and $\partial E / \partial T_{n, m}^{\mathrm{s}}=0$ used in the least-squares method, we obtain

$$
\begin{aligned}
& \frac{1}{2 \pi^{2}} \int_{0}^{2 \pi} \int_{0}^{\pi} \frac{\partial T_{m}^{\mathrm{c}, N}(\theta)}{\partial T_{n, m}^{\mathrm{c}}} \cos m \lambda R(\lambda, \theta) d \theta d \lambda=0, \\
& \frac{1}{2 \pi^{2}} \int_{0}^{2 \pi} \int_{0}^{\pi} \frac{\partial T_{m}^{\mathrm{s}, N}(\theta)}{\partial T_{n, m}^{\mathrm{s}}} \sin m \lambda R(\lambda, \theta) d \theta d \lambda=0 .
\end{aligned}
$$

From Eq. (8), we derive

$$
\frac{\partial T_{m}^{\mathrm{c}, N}(\theta)}{\partial T_{n, m}^{\mathrm{c}}}=\frac{\partial T_{m}^{\mathrm{s}, N}(\theta)}{\partial T_{n, m}^{\mathrm{s}}}=S_{n, m}(\theta),
$$

where the functions of $S_{n, m}(\theta)$ are the new DFS meridional basis functions defined as

$$
S_{n, m}(\theta) \equiv \begin{cases}\cos n \theta & \text { for } m=0, \\ \sin \theta \cos n \theta & \text { for } m=1, \\ \sin \theta \sin n \theta & \text { for even } m \geq 2, \\ \sin ^{2} \theta \sin n \theta & \text { for odd } m \geq 3 .\end{cases}
$$

Equation (27) shows that the residual $R(\lambda, \theta)$ is orthogonal to each of the new DFS basis functions $S_{m, n}(\theta) \cos m \lambda$ and $S_{m, n}(\theta) \sin m \lambda$, which means that Eq. (27) is the same as the equation derived using the Galerkin method. Thus, the equations 
$\partial E / \partial T_{n, m}^{\mathrm{c}}=0$ and $\partial E / \partial T_{n, m}^{\mathrm{s}}=0$ used in the least-squares method described in Sect. 2.3 are the same as those derived with the Galerkin method.

\subsection{Comparison of new DFS with SH}

Here we compare the new DFS method with the SH method to see the difference between them. In the SH method, $T_{m}^{\mathrm{c}}(\theta)$ and $T_{m}^{\mathrm{s}}(\theta)$ in Eq. (7) are expanded with the associated Legendre functions $P_{n, m}(\theta)$ as

$$
\begin{aligned}
& T_{m}^{\mathrm{c}}(\theta) \cong T_{m}^{\mathrm{c}, S H, N}(\theta) \equiv \sum_{n=m}^{N} T_{n, m}^{\mathrm{c}, \mathrm{SH}} P_{n, m}(\theta), \\
& T_{m}^{\mathrm{s}}(\theta) \cong T_{m}^{\mathrm{s}, S H, N}(\theta) \equiv \sum_{n=m}^{N} T_{n, m}^{\mathrm{s}, \mathrm{SH}} P_{n, m}(\theta),
\end{aligned}
$$

where $m \geq 0$. The functions $P_{n, m}(\theta)$ satisfy the following orthogonality relations for each $m$ :

$$
\int_{0}^{\pi} P_{n, m}(\theta) P_{n^{\prime}, m}(\theta) \sin \theta d \theta= \begin{cases}1 & \text { for } n=n^{\prime} \\ 0 & \text { for } n \neq n^{\prime}\end{cases}
$$

10 By the modified Robert expansion (Merilees, 1973a; Orszag, 1974), the associated Legendre functions $P_{n, m}(\theta)$ are expressed as

$$
P_{n, m}(\theta)=\sum_{\substack{l=0 \\ \text { when } n-|m|-l \text { is even }}}^{n-|m|} a_{n, m, l} \sin ^{|m|} \theta \cos l \theta .
$$

Conversely, the functions $\sin ^{|m|} \theta \cos (n-|m|) \theta \quad(n \geq|m|)$ can be expressed as the linear combination of $P_{l, m}(\theta)(l=$ $|m|, \ldots, n)$. Substituting Eq. (32) into Eq. (30) gives the following equations.

$$
\begin{aligned}
& T_{m}^{\mathrm{c}, S H, N}(\theta)=\sum_{n=0}^{N-m} T_{n, m}^{\mathrm{c}, \mathrm{SH}^{\prime}} \sin ^{m} \theta \cos n \theta, \\
& T_{m}^{\mathrm{s}, \mathrm{SH}, \mathrm{N}}(\theta)=\sum_{n=0}^{N-m} T_{n, m}^{\mathrm{s}, \mathrm{SH}^{\prime}} \sin ^{m} \theta \cos n \theta,
\end{aligned}
$$

where $m \geq 0$. Equation (33) is similar to Eq. (8) in the following sense: the basis functions for $m=0$ and $m=1 \mathrm{in} \mathrm{Eq.} \mathrm{(33)}$ are the same as in Eq. (8). The basis functions $\sin ^{2} \theta \cos n \theta(n=0, \ldots, N-2)$ for $m=2$ and $\sin ^{3} \theta \cos n \theta(n=0, \ldots, N-$ $3)$ for $m=3$ in Eq. (33) are the linear combinations of $\sin \theta \sin n \theta(n=1, \ldots, N-1)$ and $\sin ^{2} \theta \sin n \theta(n=1, \ldots, N-2)$ 20 in Eq. (8), respectively (see Eq. (A2a)), and vice versa. The basis functions for $m \geq 4$ in Eq. (33) are different from those in Eq. (8). The number of expansion coefficients in Eq. (30) or Eq. (32) in the SH method is smaller than in Eq. (8) in the new DFS method for each $m \geq 4$. From Eqs. (7) and (30), the number of expansion coefficients $T_{n, m}^{c, S H}$ in the SH model is about $N^{2} / 2$ when $M=N$. The triangular truncation used in the SH method gives a uniform resolution over the sphere. From Eqs. (7) and (8), the number of the expansion coefficients $T_{n, m}^{\mathrm{c}}$ in the DFS method is about $N^{2}$ when $M=N$. The rectangular 
truncation used in the DFS model gives almost the same resolution as the grid spacing of the regular longitude-latitude grids. Therefore, the zonal Fourier filter (see Sect. 2.11) is used in the DFS model to give a more uniform resolution.

We compare the method used to calculate the expansion coefficients in the new DFS method with that in the SH method. The SH expansion coefficients $T_{n, m}^{\mathrm{c}, \mathrm{SH}}$ and $T_{n, m}^{\mathrm{s}, \mathrm{SH}}$ in Eq. (30) are usually calculated from the grid-point values of $T_{m}^{\mathrm{c}}(\theta)$ and $5 T_{m}^{\mathrm{s}}(\theta)$, respectively, by using Gaussian quadrature or Clenshaw-Curtis quadrature (e.g., Hotta and Ujiie, 2018). They can also be calculated from $\tilde{T}_{m}^{\mathrm{c}, J}(\theta)$ and $\tilde{T}_{m}^{\mathrm{s}, J}(\theta)$ in Eq. (19) instead of $T_{m}^{\mathrm{c}}(\theta)$ and $T_{m}^{\mathrm{s}}(\theta)$ at the grid points as follows (e.g., Sneeuw and Bun, 1996):

$$
\begin{aligned}
& T_{n, m}^{\mathrm{c}, S H}=\int_{0}^{\pi} \tilde{T}_{m}^{\mathrm{c}, J}(\theta) P_{n, m}(\theta) \sin \theta d \theta, \\
& T_{n, m}^{\mathrm{s}, \mathrm{SH}}=\int_{0}^{\pi} \tilde{T}_{m}^{\mathrm{s}, J}(\theta) P_{n, m}(\theta) \sin \theta d \theta,
\end{aligned}
$$

10 where $\sin \theta$ is the latitudinal weight. The coefficients $T_{n, m}^{\mathrm{c}, \mathrm{SH}}$ and $T_{n, m}^{\mathrm{s}, \mathrm{SH}}$ can also be calculated with the least-squares method that minimizes the error $E^{\mathrm{SH}}$ (the squared $\mathrm{L}_{2}$ norm of the residual):

$$
E^{\mathrm{SH}} \equiv \frac{1}{4 \pi} \int_{0}^{2 \pi} \int_{0}^{\pi} R^{\mathrm{SH}}(\lambda, \theta)^{2} \sin \theta d \theta d \lambda,
$$

where the residual $R^{\mathrm{SH}}(\lambda, \theta)$ is

$$
R^{\mathrm{SH}}(\lambda, \theta) \equiv\left(\sum_{m=0}^{M} T_{m}^{\mathrm{c}, \mathrm{SH}, N}(\theta) \cos m \lambda+\sum_{m=1}^{M} T_{m}^{\mathrm{s}, \mathrm{SH}, N}(\theta) \sin m \lambda\right)-\left(\sum_{m=0}^{M} \tilde{T}_{m}^{\mathrm{c}, J}(\theta) \cos m \lambda+\sum_{m=1}^{M} \tilde{T}_{m}^{\mathrm{s}, J}(\theta) \sin m \lambda\right) .
$$

15 From Eqs. (35), (36), and (A3), we derive

$$
\begin{aligned}
E^{\mathrm{SH}}=\frac{1}{2} \int_{0}^{\pi}\left[\left(T_{m=0}^{\mathrm{c}, \mathrm{SH}, N}(\theta)-\widetilde{T}_{m=0}^{\mathrm{c}, \mathrm{SH}, J}(\theta)\right)^{2}\right. & +\frac{1}{2} \sum_{m=1}^{M}\left(T_{m}^{\mathrm{c}, \mathrm{SH}, N}(\theta)-\widetilde{T}_{m}^{\mathrm{c}, \mathrm{SH}, J}(\theta)\right)^{2} \\
& \left.+\frac{1}{2} \sum_{m=1}^{M}\left(T_{m}^{\mathrm{S}, \mathrm{SH}, N}(\theta)-\widetilde{T}_{m}^{\mathrm{S}, \mathrm{SH}, J}(\theta)\right)^{2}\right] \sin \theta d \theta .
\end{aligned}
$$

From Eqs. (37), (30), (31), and the equations $\partial E^{\mathrm{SH}} / \partial T_{n, m}^{\mathrm{c}, \mathrm{SH}}=0$ and $\partial E^{\mathrm{SH}} / \partial T_{n, m}^{\mathrm{s}, \mathrm{SH}}=0$ used in the least-squares method, we can derive the same equations as Eq. (34). In Eq. (35) (and Eq. (34)), the latitudinal weight $\sin \theta$ appears, unlike in Eq. (20) (and Eq. (27)), which is another difference between the SH and the new DFS methods. In the DFS method, the constant latitudinal weight is used in Eq. (20), although the latitudinal area weight described below in Appendix B is usually used as the latitudinal weight at the grid points.

When calculating the coefficients $T_{n, m}^{\mathrm{c}}$ (and $T_{n, m}^{\mathrm{s}}$ ) in Eq. (8), we can also consider the least-squares method, not using $E$ in Eq. (20) but using $E^{\prime}$ with latitudinal weight $\sin \theta$ like Eq. (35). However, minimizing $E^{\prime}$ derives the simultaneous equations for calculating $T_{n, m}^{\mathrm{c}}$ with dense matrices, which leads to $\mathrm{O}\left(N^{3}\right)$ operations. When using $E$, the simultaneous equations with penta-diagonal matrices require only $\mathrm{O}\left(N^{2}\right)$ operations. Therefore, we choose to use $E$ instead of $E^{\prime}$. 


\subsection{Application of the new basis functions to a wind vector}

The velocity potential $\chi$ and the stream function $\psi$ can be converted into the wind vector components $u$ and $v$ using the equations

$$
\begin{aligned}
& u=\frac{1}{a \cos \phi} \frac{\partial \chi}{\partial \lambda}-\frac{1}{a} \frac{\partial \psi}{\partial \phi}=\frac{1}{a \sin \theta} \frac{\partial \chi}{\partial \lambda}+\frac{1}{a} \frac{\partial \psi}{\partial \theta}, \\
& v=\frac{1}{a \cos \phi} \frac{\partial \psi}{\partial \lambda}+\frac{1}{a} \frac{\partial \chi}{\partial \phi}=\frac{1}{a \sin \theta} \frac{\partial \psi}{\partial \lambda}-\frac{1}{a} \frac{\partial \chi}{\partial \theta}
\end{aligned}
$$

where $u=a \cos \phi d \lambda / d t$ is the zonal wind, and $v=a d \phi / d t$ is the meridional wind. The scalar variables $\chi$ and $\psi$ are expanded like Eqs. (7) and (8) as

$$
\begin{aligned}
& \chi(\lambda, \theta) \cong \sum_{m=0}^{M} \chi_{m}^{\mathrm{c}}(\theta) \cos m \lambda+\sum_{m=1}^{M} \chi_{m}^{\mathrm{s}}(\theta) \sin m \lambda, \\
& \psi(\lambda, \theta) \cong \sum_{m=0}^{M} \psi_{m}^{\mathrm{c}}(\theta) \cos m \lambda+\sum_{m=1}^{M} \psi_{m}^{\mathrm{s}}(\theta) \sin m \lambda, \\
& \chi_{m}^{\mathrm{c}}(\theta) \cong \chi_{m}^{\mathrm{c}, N}(\theta) \equiv \begin{cases}\sum_{n=0}^{N} \chi_{n, m}^{\mathrm{c}} \cos n \theta & \text { for } m=0, \\
\sum_{n=0}^{N-1} \chi_{n, m}^{\mathrm{c}} \sin \theta \cos n \theta & \text { for } m=1, \\
\sum_{n=1}^{N-1} \chi_{n, m}^{\mathrm{c}} \sin \theta \sin n \theta & \text { for even } m \geq 2, \\
\sum_{n=1}^{N-2} \chi_{n, m}^{\mathrm{c}} \sin ^{2} \theta \sin n \theta & \text { for odd } m \geq 3,\end{cases} \\
& \psi_{m}^{\mathrm{c}}(\theta) \cong \psi_{m}^{\mathrm{c}, N}(\theta) \equiv \begin{cases}\sum_{n=0}^{N} \psi_{n, m}^{\mathrm{c}} \cos n \theta & \text { for } m=0, \\
\sum_{n=0}^{N-1} \psi_{n, m}^{\mathrm{c}} \sin \theta \cos n \theta & \text { for } m=1, \\
\sum_{n=1}^{N-1} \psi_{n, m}^{\mathrm{c}} \sin \theta \sin n \theta & \text { for even } m \geq 2, \\
\sum_{n=1}^{N-2} \psi_{n, m}^{\mathrm{c}} \sin ^{2} \theta \sin n \theta & \text { for odd } m \geq 3,\end{cases}
\end{aligned}
$$

The equations for $\chi_{m}^{\mathrm{s}}(\theta)$ and $\psi_{m}^{\mathrm{s}}(\theta)$ for $m \geq 1$ are the same as Eqs. (41) and (42), respectively, except that the subscript c is replaced with s. Here, the truncated variables $\psi^{N, M}(\lambda, \theta)$ and $\chi^{N, M}(\lambda, \theta)$ are defined as 


$$
\begin{aligned}
\psi^{N, M}(\lambda, \theta) & \equiv \sum_{m=0}^{M} \psi_{m}^{\mathrm{c}, N}(\theta) \cos m \lambda+\sum_{m=1}^{M} \psi_{m}^{\mathrm{s}, N}(\theta) \sin m \lambda, \\
\chi^{N, M}(\lambda, \theta) & \equiv \sum_{m=0}^{M} \chi_{m}^{\mathrm{c}, N}(\theta) \cos m \lambda+\sum_{m=1}^{M} \chi_{m}^{\mathrm{s}, N}(\theta) \sin m \lambda .
\end{aligned}
$$

The wind vector components $u^{N, M}(\lambda, \theta)$ and $v^{N, M}(\lambda, \theta)$ are obtained from $\psi^{N, M}(\lambda, \theta)$ and $\chi^{N, M}(\lambda, \theta)$ using Eq. (38) as

$$
\begin{gathered}
u^{N, M}(\lambda, \theta) \equiv \frac{1}{a \sin \theta} \frac{\partial \chi^{N, M}(\lambda, \theta)}{\partial \lambda}+\frac{1}{a} \frac{\partial \psi^{N, M}(\lambda, \theta)}{\partial \theta}=\sum_{m=0}^{M} u_{m}^{\mathrm{c}, N}(\theta) \cos m \lambda+\sum_{m=1}^{M} u_{m}^{\mathrm{s}, N}(\theta) \sin m \lambda, \\
u_{m}^{\mathrm{c}, N}(\theta) \equiv \frac{m \chi_{m}^{\mathrm{s}, N}(\theta)}{a \sin \theta}+\frac{1}{a} \frac{\partial \psi_{m}^{\mathrm{c}, N}(\theta)}{\partial \theta}, \\
u_{m}^{\mathrm{s}, N}(\theta) \equiv-\frac{m \chi_{m}^{\mathrm{c}, N}(\theta)}{a \sin \theta}+\frac{1}{a} \frac{\partial \psi_{m}^{\mathrm{s}, N}(\theta)}{\partial \theta} \\
v^{N, M}(\lambda, \theta) \equiv \frac{1}{a \sin \theta} \frac{\partial \psi^{N, M}(\lambda, \theta)}{\partial \lambda}-\frac{1}{a} \frac{\partial \chi^{N, M}(\lambda, \theta)}{\partial \theta}=\sum_{m=0}^{M} v_{m}^{\mathrm{c}, N}(\theta) \cos m \lambda+\sum_{m=1}^{M} v_{m}^{\mathrm{s}, N}(\theta) \sin m \lambda, \\
v_{m}^{\mathrm{c}, N}(\theta) \equiv \frac{m \psi_{m}^{\mathrm{s}, N}(\theta)}{a \sin \theta}-\frac{1}{a} \frac{\partial \chi_{m}^{\mathrm{c}, N}(\theta)}{\partial \theta} \\
v_{m}^{\mathrm{s}, N}(\theta) \equiv-\frac{m \psi_{m}^{\mathrm{c}, N}(\theta)}{a \sin \theta}-\frac{1}{a} \frac{\partial \chi_{m}^{\mathrm{s}, N}(\theta)}{\partial \theta} .
\end{gathered}
$$

10 From Eqs. (45b, c), (46b, c), (41), and (42), we obtain

$$
u_{m}^{\mathrm{c}, N}(\theta)=\left\{\begin{array}{lr}
\sum_{n=1}^{N} u_{n, m}^{\mathrm{c}} \sin n \theta & \text { for } m=0, \\
\sum_{n=0}^{N} u_{n, m}^{\mathrm{c}} \cos n \theta & \text { for } m=1, \\
\sum_{n=1}^{N} u_{n, m}^{\mathrm{c}} \sin n \theta & \text { for even } m \geq 2, \\
\sum_{n=0}^{N} u_{n, m}^{\mathrm{c}} \cos n \theta\left(=\sum_{n=1}^{N-1} u_{n, m}^{\mathrm{c}^{\prime}} \sin \theta \sin n \theta\right) & \text { for odd } m \geq 3,
\end{array}\right.
$$




$$
v_{m}^{\mathrm{c}, N}(\theta)=\left\{\begin{array}{lr}
\sum_{n=1}^{N} v_{n, m}^{\mathrm{c}} \sin n \theta & \text { for } m=0, \\
\sum_{n=0}^{N} v_{n, m}^{\mathrm{c}} \cos n \theta & \text { for } m=1, \\
\sum_{n=1}^{N} v_{n, m}^{\mathrm{c}} \sin n \theta & \text { for even } m \geq 2, \\
\sum_{n=0}^{N} v_{n, m}^{\mathrm{c}} \cos n \theta\left(=\sum_{n=1}^{N-1} v_{n, m}^{\mathrm{c}^{\prime}} \sin \theta \sin n \theta\right) & \text { for odd } m \geq 3,
\end{array}\right.
$$

where

$$
\begin{aligned}
& u_{n, m}^{\mathrm{c}}=-\frac{n}{a} \psi_{n, m}^{\mathrm{c}} \quad \text { for } m=0, \\
& u_{n, m}^{\mathrm{c}}=\frac{1}{a}\left[m \chi_{n, m}^{\mathrm{s}}+\frac{n\left(\psi_{n-1, m}^{\mathrm{c}}-\psi_{n+1, m}^{\mathrm{c}}\right)}{2}\right] \quad \text { for } m=1 \text {, } \\
& \text { except for } u_{1, m}^{\mathrm{c}}=\frac{1}{a}\left[m \chi_{1, m}^{\mathrm{s}}+\frac{2 \psi_{0, m}^{\mathrm{c}}-\psi_{2, m}^{\mathrm{c}}}{2}\right] \text {, } \\
& u_{n, m}^{\mathrm{c}}=\frac{1}{a}\left[m \chi_{n, m}^{\mathrm{s}}+\frac{n\left(\psi_{n-1, m}^{\mathrm{c}}-\psi_{n+1, m}^{\mathrm{c}}\right)}{2}\right] \quad \text { for even } m \geq 2 \text {, } \\
& u_{n, m}^{\mathrm{c}}=\frac{1}{a}\left[\frac{m\left(-\chi_{n-1, m}^{\mathrm{s}}+\chi_{n+1, m}^{\mathrm{s}}\right)}{2}+\frac{n\left(-\psi_{n-2, m}^{\mathrm{c}}+2 \psi_{n, m}^{\mathrm{c}}-\psi_{n+2, m}^{\mathrm{c}}\right)}{4}\right] \text { for odd } m \geq 3 \\
& \text { except for } u_{1, m}^{\mathrm{c}}=\frac{1}{a}\left[\frac{m \chi_{2, m}^{\mathrm{s}}}{2}+\frac{\left(3 \psi_{1, m}^{\mathrm{c}}-\psi_{3, m}^{\mathrm{c}}\right)}{4}\right] \text {, }
\end{aligned}
$$

The equations for $u_{m}^{\mathrm{s}, N}(\theta)$ and $v_{m}^{\mathrm{s}, N}(\theta)$ for $m \geq 1$ are the same as Eqs. (47) and (48), respectively, except that the subscript

$10 \mathrm{c}$ is replaced with s. The equations for $u_{n, m}^{\mathrm{s}}$ are the same as Eqs. $(49 \mathrm{~b}, \mathrm{c}, \mathrm{d})$, except that $u_{n, m}^{\mathrm{c}}, \chi_{n, m}^{\mathrm{s}}$, and $\psi_{n, m}^{\mathrm{c}}$ are replaced with $u_{n, m}^{\mathrm{s}},-\chi_{n, m}^{\mathrm{c}}$, and $\psi_{n, m}^{\mathrm{s}}$, respectively. The equations for $v_{n, m}^{\mathrm{c}}$ are the same as Eqs. (49a, b, c, d), except that $u_{n, m}^{\mathrm{c}}, \chi_{n, m}^{\mathrm{s}}$, and $\psi_{n, m}^{\mathrm{c}}$ are replaced with $v_{n, m}^{\mathrm{c}}, \psi_{n, m}^{\mathrm{s}}$, and $-\chi_{n, m}^{\mathrm{c}}$, respectively. The equations for $v_{n, m}^{\mathrm{s}}$ are the same as Eqs. $(49 \mathrm{~b}, \mathrm{c}, \mathrm{d})$, except that $u_{n, m}^{\mathrm{c}}, \chi_{n, m}^{\mathrm{s}}$, and $\psi_{n, m}^{\mathrm{c}}$ are replaced with $v_{n, m}^{\mathrm{s}},-\psi_{n, m}^{\mathrm{c}}$, and $-\chi_{n, m}^{\mathrm{s}}$, respectively.

From Eqs. (47) to (49), it can be seen that $u_{m}^{\mathrm{c}, N}(\theta), u_{m}^{\mathrm{s}, N}(\theta), v_{m}^{\mathrm{c}, N}(\theta)$, and $v_{m}^{\mathrm{s}, N}(\theta)$ at the poles are finite for $m=1$ and zero 15 for $m \neq 1$. Moreover, the following relations are satisfied for $m=1$ :

$$
\begin{aligned}
& u_{m=1}^{\mathrm{c}, N}(\theta)=-v_{m=1}^{\mathrm{s}, N}(\theta)\left(=\frac{1}{a} \sum_{n=1}^{N-1}\left(\chi_{n, m=1}^{\mathrm{s}}+\psi_{n, m=1}^{\mathrm{c}}\right)\right) \quad \text { at } \theta=0 \text { (North Pole), } \\
& u_{m=1}^{\mathrm{s}, N}(\theta)=v_{m=1}^{\mathrm{c}, N}(\theta)\left(=\frac{1}{a} \sum_{n=1}^{N-1}\left(-\chi_{n, m=1}^{\mathrm{c}}+\psi_{n, m=1}^{\mathrm{s}}\right)\right) \quad \text { at } \theta=0 \text { (North Pole), }
\end{aligned}
$$




$$
\begin{aligned}
& u_{m=1}^{\mathrm{c}, N}(\theta)=v_{m=1}^{\mathrm{s}, N}(\theta)\left(=\frac{1}{a} \sum_{n=0}^{N-1}(-1)^{n}\left(\chi_{n, m=1}^{\mathrm{s}}+\psi_{n, m=1}^{\mathrm{c}}\right)\right) \text { at } \theta=\pi \text { (South Pole), } \\
& u_{m=1}^{\mathrm{s}, N}(\theta)=-v_{m=1}^{\mathrm{c}, N}(\theta)\left(=\frac{1}{a} \sum_{n=0}^{N-1}(-1)^{n}\left(-\chi_{n, m=1}^{\mathrm{c}}+\psi_{n, m=1}^{\mathrm{s}}\right)\right) \text { at } \theta=\pi \text { (South Pole). }
\end{aligned}
$$

Thus, it is guaranteed that the wind vector $\left(u^{N, M}, v^{N, M}\right)$ in Eqs. (45) and (46) is continuous at the poles.

\subsection{New method to calculate expansion coefficients for a wind vector}

We propose a new method that calculates the expansion coefficients $\chi_{n, m}^{\mathrm{c}}, \chi_{n, m}^{\mathrm{s}}, \psi_{n, m}^{\mathrm{c}}$ and $\psi_{n, m}^{\mathrm{s}}$ using the least-squares method to minimize the error of $u^{N, M}(\lambda, \theta)$ and $v^{N, M}(\lambda, \theta)$ with respect to $u(\lambda, \theta)$ and $v(\lambda, \theta)$ due to the meridional wavenumber truncation. First, the wind vector components $u$ and $v$ are expanded as

$$
\begin{aligned}
& u(\lambda, \theta) \cong \sum_{m=0}^{M} u_{m}^{\mathrm{c}}(\theta) \cos m \lambda+\sum_{m=1}^{M} u_{m}^{\mathrm{s}}(\theta) \sin m \lambda, \\
& v(\lambda, \theta) \cong \sum_{m=0}^{M} v_{m}^{\mathrm{c}}(\theta) \cos m \lambda+\sum_{m=1}^{M} v_{m}^{\mathrm{s}}(\theta) \sin m \lambda, \text { for even } m, \\
& u_{m}^{\mathrm{c}}(\theta) \cong \tilde{u}_{m}^{\mathrm{c}, J}(\theta) \equiv \begin{cases}\sum_{n=1}^{J} \tilde{u}_{n, m}^{\mathrm{c}} \sin n \theta & \text { for odd } m, \\
\sum_{n=0}^{J-1} \tilde{u}_{n, m}^{\mathrm{c}} \cos n \theta & \text { for even } m,\end{cases} \\
& v_{m}^{\mathrm{c}}(\theta) \cong \tilde{v}_{m}^{\mathrm{c}, J}(\theta) \equiv \begin{cases}J \\
\sum_{n=1}^{J} \tilde{v}_{n, m}^{\mathrm{c}} \sin n \theta, & \text { for odd } m . \\
\sum_{n=0}^{J-1} \tilde{v}_{n, m}^{\mathrm{c}} \cos n \theta, & \end{cases}
\end{aligned}
$$

The equations for $u_{m}^{\mathrm{s}}(\theta)$ and $v_{m}^{\mathrm{s}}(\theta)$ for $m \geq 1$ are the same as Eqs. (53) and (54), respectively, except that the subscript c is replaced with s. Here the expansion coefficients $\tilde{u}_{n, m}^{\mathrm{c}}, \tilde{u}_{n, m}^{\mathrm{s}}, \tilde{v}_{n, m}^{\mathrm{c}}$, and $\tilde{v}_{n, m}^{\mathrm{s}}$ are calculated by the forward discrete cosine or sine transform from the grid-point values of $u_{m}^{\mathrm{c}}(\theta), u_{m}^{\mathrm{s}}(\theta), v_{m}^{\mathrm{c}}(\theta)$, and $v_{m}^{\mathrm{s}}(\theta)$ (See Sect. 2.10). The truncated variables $15 \tilde{u}^{J, M}(\lambda, \theta)$ and $\tilde{v}^{J, M}(\lambda, \theta)$ are defined as

$$
\begin{aligned}
& \tilde{u}^{J, M}(\lambda, \theta) \equiv \sum_{m=0}^{M} \tilde{u}_{m}^{\mathrm{c} J}(\theta) \cos m \lambda+\sum_{m=1}^{M} \tilde{u}_{m}^{\mathrm{s}, J}(\theta) \sin m \lambda \\
& \tilde{v}^{J, M}(\lambda, \theta) \equiv \sum_{m=0}^{M} \tilde{v}_{m}^{\mathrm{c}, J}(\theta) \cos m \lambda+\sum_{m=1}^{M} \tilde{v}_{m}^{\mathrm{s}, J}(\theta) \sin m \lambda .
\end{aligned}
$$


Next, $\chi_{n, m}^{\mathrm{c}}, \chi_{n, m}^{\mathrm{s}}, \psi_{n, m}^{\mathrm{c}}$, and $\psi_{n, m}^{\mathrm{s}}$ are calculated to minimize the following error $F$ (the squared $\mathrm{L}_{2}$ norm of the residual vector):

$$
F \equiv \frac{1}{2 \pi^{2}} \int_{0}^{2 \pi} \int_{0}^{\pi}\left(R_{n, m}^{u}(\lambda, \theta)^{2}+R_{n, m}^{v}(\lambda, \theta)^{2}\right) d \theta d \lambda,
$$

where the residual vector $\left(R_{n, m}^{u}(\lambda, \theta), R_{n, m}^{v}(\lambda, \theta)\right)$ is defined as

5

10

15

$$
\begin{aligned}
& R_{n, m}^{u}(\lambda, \theta) \equiv u^{N, M}(\lambda, \theta)-\tilde{u}^{J, M}(\lambda, \theta), \\
& R_{n, m}^{v}(\lambda, \theta) \equiv v^{N, M}(\lambda, \theta)-\tilde{v}^{J, M}(\lambda, \theta) .
\end{aligned}
$$

From Eqs. (55) to (58) and Eqs. (45a), (46a), and (A3), we derive

$$
\begin{aligned}
F= & \frac{1}{\pi} \int_{0}^{\pi}\left\{\left[\left(u_{m=0}^{\mathrm{c}, N}(\theta)-\tilde{u}_{m=0}^{\mathrm{c}, J}(\theta)\right)+\frac{1}{2} \sum_{m=1}^{M}\left(u_{m}^{\mathrm{c}, N}(\theta)-\tilde{u}_{m}^{\mathrm{c}, J}(\theta)\right)\right]^{2}+\frac{1}{2}\left[\sum_{m=1}^{M}\left(u_{m}^{\mathrm{s}, N}(\theta)-\tilde{u}_{m}^{\mathrm{s}, J}(\theta)\right)\right]^{2}\right. \\
& \left.+\left[\left(v_{m=0}^{\mathrm{c}, N}(\theta)-\tilde{v}_{m=0}^{\mathrm{c}, J}(\theta)\right)+\frac{1}{2} \sum_{m=1}^{M}\left(v_{m}^{\mathrm{c}, N}(\theta)-\tilde{v}_{m}^{\mathrm{c}, J}(\theta)\right)\right]^{2}+\frac{1}{2}\left[\sum_{m=1}^{M}\left(v_{m}^{\mathrm{s}, N}(\theta)-\tilde{v}_{m}^{\mathrm{s}, J}(\theta)\right)\right]^{2}\right\} d \theta
\end{aligned}
$$

Equations (47), (48), (49), (53), and (54) are substituted into Eq. (59). The equations $\partial F / \partial \chi_{m, n}^{\mathrm{c}}=0, \partial F / \partial \chi_{n, m}^{\mathrm{s}}=0$, $\partial F / \partial \psi_{n, m}^{\mathrm{c}}=0$, and $\partial F / \partial \psi_{n, m}^{\mathrm{s}}=0$ are used to calculate $\chi_{n, m}^{\mathrm{c}}, \chi_{n, m}^{\mathrm{s}}, \psi_{n, m}^{\mathrm{c}}$, and $\psi_{n, m}^{\mathrm{s}}$, so that $F$ is minimized.

From $\partial F / \partial \chi_{m, n}^{c}=0$ and Eq. (A4), we derive

$$
\begin{array}{cc}
\frac{\partial}{\partial \chi_{m, n}^{\mathrm{c}}}\left[\sum_{n=1}^{N}\left(v_{n, m}^{\mathrm{c}}-\tilde{v}_{n, m}^{\mathrm{c}}\right)^{2}\right]=0 & \text { for } m=0, \\
\frac{\partial}{\partial \chi_{m, n}^{\mathrm{c}}}\left[\sum_{n=1}^{N}\left(u_{n, m}^{\mathrm{s}}-\tilde{u}_{n, m}^{\mathrm{s}}\right)^{2}+\sum_{n=1}^{N}\left(v_{n, m}^{\mathrm{c}}-\tilde{v}_{n, m}^{\mathrm{c}}\right)^{2}\right]=0 & \text { for even } m \geq 2, \\
\frac{\partial}{\partial \chi_{m, n}^{\mathrm{c}}}\left[2\left(u_{0, m}^{\mathrm{s}}-\tilde{u}_{0, m}^{\mathrm{s}}\right)^{2}+\sum_{n=1}^{N}\left(u_{n, m}^{\mathrm{s}}-\tilde{u}_{n, m}^{\mathrm{s}}\right)^{2}+2\left(v_{0, m}^{\mathrm{c}}-\tilde{v}_{0, m}^{\mathrm{c}}\right)^{2}+\sum_{n=1}^{N}\left(v_{n, m}^{\mathrm{c}}-\tilde{v}_{n, m}^{\mathrm{c}}\right)^{2}\right]=0 & \text { for odd } m .
\end{array}
$$

From Eq. (60), and from the same equations as Eqs. (49b,c,d), except that $u_{n, m}^{\mathrm{c}}, \chi_{n, m}^{\mathrm{s}}$, and $\psi_{n, m}^{\mathrm{c}}$ are replaced with $u_{n, m}^{\mathrm{s}}$, $-\chi_{n, m}^{\mathrm{c}}$, and $\psi_{n, m}^{\mathrm{s}}$, respectively, and the same equations as Eqs. $(49 \mathrm{a}, \mathrm{b}, \mathrm{c}, \mathrm{d})$, except that $u_{n, m}^{\mathrm{c}}, \chi_{n, m}^{\mathrm{s}}$, and $\psi_{n, m}^{\mathrm{c}}$ are replaced with $v_{n, m}^{\mathrm{c}}, \psi_{n, m}^{\mathrm{s}}$, and $-\chi_{n, m}^{\mathrm{c}}$, respectively, we derive the following equations for $\chi_{n, m}^{\mathrm{c}}$ and $\psi_{n, m}^{\mathrm{s}}$.

For $m=0$,

$$
\frac{1}{a}\left[n \chi_{m, n}^{\mathrm{c}}\right]=\tilde{v}_{m, n}^{\mathrm{c}} \quad(1 \leq n \leq N)
$$

The coefficient $\chi_{m=0, n=0}^{\mathrm{c}}$ is determined so that the global means of $\chi$ are zero. See Appendix B for the calculation of the global mean.

For $m=1$, 


$$
\begin{aligned}
\frac{1}{a}\left[-(n-1)^{2} \chi_{n-2, m}^{\mathrm{c}}-\right. & \left.2 m \psi_{n-1, m}^{\mathrm{s}}+\left(4 m^{2}+2 n^{2}+2\right) \chi_{n, m}^{\mathrm{c}}-2 m \psi_{n+1, m}^{\mathrm{s}}-(n+1)^{2} \chi_{n+2, m}^{\mathrm{c}}\right] \\
= & 2(n-1) \tilde{v}_{n-1, m}^{\mathrm{c}}-4 m \tilde{u}_{n, m}^{\mathrm{s}}-2(n+1) \tilde{v}_{n+1, m}^{\mathrm{c}}(0 \leq n \leq N-1),
\end{aligned}
$$

with the exception of the following underlined values:

5

For even $m \geq 2$,

$$
\begin{array}{cc}
\frac{1}{a}\left[\left(\underline{8} m^{2}+\underline{4}\right) \chi_{0, m}^{\mathrm{c}}-\underline{4} m \psi_{1, m}^{\mathrm{s}}-\underline{2} \chi_{2, m}^{\mathrm{c}}\right]=-\underline{8} m \tilde{u}_{0, m}^{\mathrm{s}}-\underline{4}_{1, m}^{\mathrm{c}} & (n=0), \\
\frac{1}{a}\left[-\underline{4} m \psi_{0, m}^{\mathrm{s}}+\left(4 m^{2}+4\right) \chi_{1, m}^{\mathrm{c}}+\cdots\right]=\cdots & (n=1), \\
\frac{1}{a}\left[-\underline{2} \chi_{0, m}^{\mathrm{c}}-2 m \psi_{1, m}^{\mathrm{s}}+\cdots\right]=\cdots & (n=2),
\end{array}
$$

$$
\begin{aligned}
\frac{1}{a}\left[-(n-1)^{2} \chi_{n-2, m}^{\mathrm{c}}-\right. & \left.2 m \psi_{n-1, m}^{\mathrm{s}}+\left(4 m^{2}+2 n^{2}+2\right) \chi_{n, m}^{\mathrm{c}}-2 m \psi_{n+1, m}^{\mathrm{s}}-(n+1)^{2} \chi_{n+2, m}^{\mathrm{c}}\right] \\
= & 2(n-1) \tilde{v}_{n-1, m}^{\mathrm{c}}-4 m \tilde{u}_{n, m}^{\mathrm{s}}-2(n+1) \tilde{v}_{n+1, m}^{\mathrm{c}} \quad(1 \leq n \leq N-1),
\end{aligned}
$$

10 with no exception.

For odd $m \geq 3$,

$$
\begin{array}{r}
\frac{1}{a}\left[(n-2)^{2} \chi_{n-4, m}^{\mathrm{c}}+2 m \psi_{n-3, m}^{\mathrm{s}}+\left(-4 m^{2}-4 n^{2}+8 n-8\right) \chi_{n-2, m}^{\mathrm{c}}-2 m \psi_{n-1, m}^{\mathrm{s}}+\left(8 m^{2}+6 n^{2}+8\right) \chi_{n, m}^{\mathrm{c}}\right. \\
\left.-2 m \psi_{n+1, m}^{\mathrm{s}}+\left(-4 m^{2}-4 n^{2}-8 n-8\right) \chi_{n+2, m}^{\mathrm{c}}+2 m \psi_{n+3, m}^{\mathrm{s}}+(n+2)^{2} \chi_{n+4, m}^{\mathrm{c}}\right] \\
=4(n-2) \tilde{v}_{n-2, m}^{\mathrm{c}}-8 m \tilde{u}_{n-1, m}^{\mathrm{s}}-8 n \tilde{v}_{n, m}^{\mathrm{c}}+8 m \tilde{u}_{n+1, m}^{\mathrm{s}}+4(n+2) \tilde{v}_{n+2, m}^{\mathrm{c}} \quad(1 \leq n \leq N-2),
\end{array}
$$

with the exception of the following underlined values:

$$
\begin{array}{cc}
\frac{1}{a}\left[\left(\underline{12} m^{2}+\underline{18}\right) \chi_{1, m}^{\mathrm{c}}-\underline{4} m \psi_{2, m}^{\mathrm{s}}+\left(-4 m^{2}-\underline{21}\right) \chi_{3, m}^{\mathrm{c}}+\cdots\right]=-\underline{16} m \tilde{u}_{0, m}^{\mathrm{s}}-\underline{12} \tilde{v}_{1, m}^{\mathrm{c}}+\cdots & (n=1), \\
\frac{1}{a}\left[-\underline{4} m \psi_{1, m}^{\mathrm{s}}+\left(8 m^{2}+32\right) \chi_{2, m}^{\mathrm{c}}+\cdots\right]=\cdots & (n=2), \\
\frac{1}{a}\left[\left(-4 m^{2}-\underline{21}\right) \chi_{1, m}^{\mathrm{c}}-2 m \psi_{2, m}^{\mathrm{s}}+\cdots\right]=\cdots & (n=3) .
\end{array}
$$

Similarly, from $\partial F / \partial \chi_{m, n}^{\mathrm{s}}=0$, we derive the same equations as Eqs. $(61 \mathrm{~b}, \mathrm{c}, \mathrm{d})$, except that $\chi^{\mathrm{c}}, \psi^{\mathrm{s}}, \tilde{v}^{\mathrm{c}}$, and $\tilde{u}^{\mathrm{s}}$ are replaced with $\chi^{\mathrm{s}},-\psi^{\mathrm{c}}, \tilde{v}^{\mathrm{s}}$, and $-\tilde{u}^{\mathrm{c}}$, respectively. From $-\partial F / \partial \psi_{m, n}^{\mathrm{c}}=0$, we derive the same equations as Eqs. (61a, b, c, d), except that $\chi^{\mathrm{c}}, \psi^{\mathrm{s}}, \tilde{v}^{\mathrm{c}}$, and $\tilde{u}^{\mathrm{s}}$ are replaced with $-\psi^{\mathrm{c}}, \chi^{\mathrm{s}}, \tilde{u}^{\mathrm{c}}$, and $-\tilde{v}^{\mathrm{s}}$, respectively. From $\partial F / \partial \psi_{m, n}^{\mathrm{s}}=0$, we derive the same equations as Eqs. (61b,c,d), except that $\chi^{\mathrm{c}}, \psi^{\mathrm{s}}, \tilde{v}^{\mathrm{c}}$, and $\tilde{u}^{\mathrm{s}}$ are replaced with $\psi^{\mathrm{s}}, \chi^{\mathrm{c}},-\tilde{u}^{\mathrm{s}}$, and $-\tilde{v}^{\mathrm{c}}$, respectively.

From Eqs. (61b, c, d), and from the same equations as Eqs. $(61 \mathrm{~b}, \mathrm{c}, \mathrm{d})$, except that $\chi^{\mathrm{c}}, \psi^{\mathrm{s}}, \tilde{v}^{\mathrm{c}}$, and $\tilde{u}^{\mathrm{s}}$ are replaced with $\psi^{\mathrm{s}}$, $\chi^{\mathrm{c}},-\tilde{u}^{\mathrm{s}}$, and $-\tilde{v}^{\mathrm{c}}$, respectively, we derive the following two linear simultaneous equations with nine-diagonal matrices for each odd $m \geq 3$ : 


$$
\mathbf{D}_{1}\left[\begin{array}{c}
\chi_{0, m}^{\mathrm{c}} \\
\psi_{1, m}^{\mathrm{s}} \\
\chi_{2, m}^{\mathrm{c}} \\
\psi_{3, m}^{\mathrm{s}} \\
\chi_{4, m}^{\mathrm{c}} \\
\psi_{5, m}^{\mathrm{s}} \\
\vdots \\
:
\end{array}\right]=\left[\begin{array}{c}
* \\
* \\
* \\
* \\
* \\
* \\
: \\
*
\end{array}\right], \quad \mathbf{D}_{2}\left[\begin{array}{c}
\psi_{0, m}^{\mathrm{s}} \\
\chi_{1, m}^{\mathrm{c}} \\
\psi_{2, m}^{\mathrm{s}} \\
\chi_{3, m}^{\mathrm{c}} \\
\psi_{4, m}^{\mathrm{s}} \\
\chi_{5, m}^{\mathrm{c}} \\
\vdots \\
:
\end{array}\right]=\left[\begin{array}{c}
* \\
* \\
* \\
* \\
* \\
* \\
: \\
*
\end{array}\right],
$$

where $\mathbf{D}_{\mathbf{1}}$ and $\mathbf{D}_{\mathbf{2}}$ are nine-diagonal matrices. We also derive two similar linear simultaneous equations with penta-diagonal matrices for $m=1$ and each even $m \geq 2$. The simultaneous equations with nine-diagonal or penta-diagonal matrices can be solved in a similar way to Eq. (25), and the expansion coefficients $\chi_{n, m}^{\mathrm{c}}$ and $\psi_{n, m}^{\mathrm{s}}$ in Eq. (62) can be solved efficiently. From

5 the same equations as Eqs. $(61 \mathrm{~b}, \mathrm{c}, \mathrm{d})$, except that $\chi^{\mathrm{c}}, \psi^{\mathrm{s}}, \tilde{v}^{\mathrm{c}}$, and $\tilde{u}^{s}$ are replaced with $\chi^{\mathrm{s}},-\psi^{\mathrm{c}}, \tilde{v}^{\mathrm{s}}$, and $-\tilde{u}^{\mathrm{c}}$, respectively, and the same equations as Eqs. $(61 \mathrm{~b}, \mathrm{c}, \mathrm{d})$, except that $\chi^{\mathrm{c}}, \psi^{\mathrm{s}}, \tilde{v}^{\mathrm{c}}$, and $\tilde{u}^{\mathrm{s}}$ are replaced with $-\psi^{\mathrm{c}}, \chi^{\mathrm{s}}, \tilde{u}^{\mathrm{c}}$, and $-\tilde{v}^{\mathrm{s}}$, respectively, two similar linear simultaneous equations with nine-diagonal matrices for each $m \geq 3$ and two linear simultaneous equations with penta-diagonal matrices for $m=1$ and each even $m \geq 2$ are also derived. Thus, the expansion coefficients $\chi_{n, m}^{\mathrm{c}}, \chi_{n, m}^{\mathrm{s}}$, $\psi_{n, m}^{\mathrm{c}}$, and $\psi_{n, m}^{\mathrm{s}}$ are obtained from $\tilde{u}_{n, m}^{\mathrm{c}}, \tilde{u}_{n, m}^{\mathrm{s}}, \tilde{v}_{n, m}^{\mathrm{c}}$, and $\tilde{v}_{n, m}^{\mathrm{s}}$ using Eqs. $(61 \mathrm{a}, \mathrm{b}, \mathrm{c}, \mathrm{d})$ and the similar equations.

The expansion coefficients $u_{n, m}^{\mathrm{c}}, u_{n, m}^{\mathrm{s}}, v_{n, m}^{\mathrm{c}}$, and $v_{n, m}^{\mathrm{s}}$ are obtained from $\chi_{n, m}^{\mathrm{c}}, \chi_{n, m}^{\mathrm{s}}, \psi_{n, m}^{\mathrm{c}}$, and $\psi_{n, m}^{s}$ using Eq. (49) for $u_{n, m}^{\mathrm{c}}$ and the similar equations for $u_{n, m}^{\mathrm{s}}, v_{n, m}^{\mathrm{c}}$, and $v_{n, m}^{\mathrm{s}}$.

\subsection{Relation between the least-squares method and the Galerkin method for the wind vector}

Here we discuss the relation between the least-squares method described above and the Galerkin method when calculating the expansion coefficients related to the wind vector.

From Eqs. (57), (58), and the equations $\partial F / \partial \chi_{m, n}^{\mathrm{c}}=0, \partial F / \partial \chi_{n, m}^{\mathrm{s}}=0, \partial F / \partial \psi_{n, m}^{\mathrm{c}}=0$, and $\partial F / \partial \psi_{n, m}^{\mathrm{s}}=0$ used in the least-squares method, we obtain

$$
\begin{aligned}
& \frac{1}{2 \pi^{2}} \int_{0}^{2 \pi} \int_{0}^{\pi}\left[\frac{\partial u^{N, M}(\lambda, \theta)}{\partial \chi_{n, m}^{\mathrm{c}}} R_{n, m}^{u}(\lambda, \theta)+\frac{\partial v^{N, M}(\lambda, \theta)}{\partial \chi_{n, m}^{\mathrm{c}}} R_{n, m}^{v}(\lambda, \theta)\right] d \theta d \lambda=0, \\
& \frac{1}{2 \pi^{2}} \int_{0}^{2 \pi} \int_{0}^{\pi}\left[\frac{\partial u^{N, M}(\lambda, \theta)}{\partial \chi_{n, m}^{\mathrm{s}}} R_{n, m}^{u}(\lambda, \theta)+\frac{\partial v^{N, M}(\lambda, \theta)}{\partial \chi_{n, m}^{\mathrm{s}}} R_{n, m}^{v}(\lambda, \theta)\right] d \theta d \lambda=0, \\
& \frac{1}{2 \pi^{2}} \int_{0}^{2 \pi} \int_{0}^{\pi}\left[\frac{\partial u^{N, M}(\lambda, \theta)}{\partial \psi_{n, m}^{\mathrm{c}}} R_{n, m}^{u}(\lambda, \theta)+\frac{\partial v^{N, M}(\lambda, \theta)}{\partial \psi_{n, m}^{\mathrm{c}}} R_{n, m}^{v}(\lambda, \theta)\right] d \theta d \lambda=0, \\
& \frac{1}{2 \pi^{2}} \int_{0}^{2 \pi} \int_{0}^{\pi}\left[\frac{\partial u^{N, M}(\lambda, \theta)}{\partial \psi_{n, m}^{\mathrm{s}}} R_{n, m}^{u}(\lambda, \theta)+\frac{\partial v^{N, M}(\lambda, \theta)}{\partial \psi_{n, m}^{\mathrm{s}}} R_{n, m}^{v}(\lambda, \theta)\right] d \theta d \lambda=0 .
\end{aligned}
$$

From Eqs. (45), (46), and (28) we derive

$$
\left(\frac{\partial u^{N, M}(\lambda, \theta)}{\partial \chi_{n, m}^{\mathrm{c}}}, \frac{\partial v^{N, M}(\lambda, \theta)}{\partial \chi_{n, m}^{\mathrm{c}}}\right)=\left(\frac{\partial}{\partial \chi_{n, m}^{\mathrm{c}}}\left(-\frac{m \chi_{m}^{\mathrm{c}, N}(\theta)}{a \sin \theta} \sin m \lambda\right), \frac{\partial}{\partial \chi_{n, m}^{\mathrm{c}}}\left(-\frac{1}{a} \frac{\partial \chi_{m}^{\mathrm{c}, N}(\theta)}{\partial \theta} \cos m \lambda\right)\right)
$$




$$
\begin{aligned}
& =\left(-\frac{m S_{n, m}(\theta)}{a \sin \theta} \sin m \lambda,-\frac{1}{a} \frac{\partial S_{n, m}(\theta)}{\partial \theta} \cos m \lambda\right), \\
\left(\frac{\partial u^{N, M}(\lambda, \theta)}{\partial \chi_{n, m}^{\mathrm{s}}}, \frac{\partial v^{N, M}(\lambda, \theta)}{\partial \chi_{n, m}^{\mathrm{s}}}\right) & =\left(\frac{m S_{n, m}(\theta)}{a \sin \theta} \cos m \lambda,-\frac{1}{a} \frac{\partial S_{n, m}(\theta)}{\partial \theta} \sin m \lambda\right), \\
\left(\frac{\partial u^{N, M}(\lambda, \theta)}{\partial \psi_{n, m}^{\mathrm{c}}}, \frac{\partial v^{N, M}(\lambda, \theta)}{\partial \psi_{n, m}^{\mathrm{c}}}\right) & =\left(\frac{1}{a} \frac{\partial S_{n, m}(\theta)}{\partial \theta} \cos m \lambda,-\frac{m S_{n, m}(\theta)}{a \sin \theta} \sin m \lambda\right), \\
\left(\frac{\partial u^{N, M}(\lambda, \theta)}{\partial \psi_{n, m}^{\mathrm{s}}}, \frac{\partial v^{N, M}(\lambda, \theta)}{\partial \psi_{n, m}^{\mathrm{s}}}\right) & =\left(\frac{1}{a} \frac{\partial S_{n, m}(\theta)}{\partial \theta} \sin m \lambda, \frac{m S_{n, m}(\theta)}{a \sin \theta} \cos m \lambda\right) .
\end{aligned}
$$

5 The right-hand sides of Eqs. (64a, b, c, d) are considered as the new DFS vector basis functions. Equation (63) shows that the residual vector $\left(R_{n, m}^{u}(\lambda, \theta), R_{n, m}^{v}(\lambda, \theta)\right)$ is orthogonal to each of the vector basis functions in Eq. (64), which means that Eq. (63) is the same as the equation obtained by the Galerkin method.

This method to calculate the DFS expansion coefficients of $\chi$ and $\psi$ from $u$ and $v$ using the least-squares method (or the Galerkin method with the DFS vector basis functions) is similar to the vector harmonic transform method (Browning et al., 1989; Temperton, 1991; Swarztrauber, 1993), where the SH expansion coefficients of the divergence $D=\nabla^{2} \chi$ and the vorticity $\zeta=\nabla^{2} \psi$ are calculated from the grid-point values of $u$ and $v$ using the Galerkin spectral method with the orthogonal vector $\mathrm{SH}$ basis functions.

\subsection{Arrangement of equally spaced latitudinal grid points}

In DFS models, equally spaced latitudinal grid points are used. We use the following three ways of arranging equally spaced latitudinal grid points:

$$
\begin{aligned}
& \text { Grid [0] : } J=J^{0}, \quad \theta_{j}=\pi(j+0.5) / J^{0}, \quad j=0, \ldots, J^{0}-1 \text {, } \\
& \text { Grid [1] : } J=J^{0}+1, \quad \theta_{j}=\pi j / J^{0}, \quad j=0, \ldots, J^{0} \text {, } \\
& \text { Grid [-1] : } J=J^{0}-1, \theta_{j}=\pi j / J^{0}, \quad j=1, \ldots, J^{0}-1,
\end{aligned}
$$

where $\theta_{j}$ is the latitude at each grid point, and $J^{0}$ is the number of latitudinal grid points in Grid [0]. When the grid intervals in Grids [0], [1], and [-1] are set equal, the number of grid points $J$ in Grid [1] is $J^{0}+1$ and the number of grid points $J$ in Grid [ -1$]$ is $J^{0}-1$. Figure 1 shows Grids [0], [1], and [-1] when $J^{0}=4$ and the grid interval $\Delta \theta=\pi / 4$. Grid [0] has been widely used in DFS models, for example, in Merilees (1973b), Orszag (1974), Cheong (2000a, 2000b), and Yoshimura and Matsumura (2005). Grid [1] was used, for example, in the DFS expansion in Yee (1981). Grid [-1] was used, for example, in the SH model using Clenshaw-Curtis quadrature in Hotta and Ujiie (2018). All of Grids [0], [1], and [-1] were used in the SH expansion in Swarztrauber and Spotz (2000). We have confirmed that stable integration is possible in the model using the new DFS method with any of Grids [0], [1], and [-1], as shown in Sect. 5 below. 


\subsection{Discrete Fourier cosine and sine transforms in latitude}

Forward discrete Fourier cosine and sine transforms are performed in Eqs. (19), (53), and (54), and inverse discrete Fourier cosine and sine transforms are performed in Eqs. (9), (47), and (48), in the latitudinal direction. The calculation of the discrete cosine and sine transforms in Grids [0], [1], and [-1] is shown below. Here, $g\left(\theta_{j}\right)$ and $h\left(\theta_{j}\right)$ are grid-point values, 5 and $g_{n}$ and $h_{n}$ are expansion coefficients.

When using Grid [0], forward and inverse discrete cosine transforms are performed as

$$
\begin{aligned}
& g_{n}=\frac{b}{J^{0}} \sum_{j=0}^{J^{0}-1} g\left(\theta_{j}\right) \cos n \theta_{j}, \quad b \equiv\left\{\begin{array}{l}
1 \text { for } n=0 \\
2 \text { for } n \neq 0,
\end{array}\right. \\
& g\left(\theta_{j}\right)=\sum_{n=0}^{J^{0}-1} g_{n} \cos n \theta_{j} .
\end{aligned}
$$

When using Grid [0], forward and inverse discrete sine transforms are performed as

$$
\begin{aligned}
& h_{n}=\frac{b}{J^{0}} \sum_{j=0}^{J^{0}-1} h\left(\theta_{j}\right) \sin n \theta_{j}, \quad b \equiv\left\{\begin{array}{l}
1 \text { for } n=J^{0} \\
2 \text { for } n \neq J^{0},
\end{array}\right. \\
& h\left(\theta_{j}\right)=\sum_{n=1}^{J^{0}} h_{n} \sin n \theta_{j} .
\end{aligned}
$$

When using Grid [1], forward and inverse discrete cosine transforms are performed as

$$
\begin{aligned}
& g_{n}=\frac{b}{J^{0}} \sum_{j=0}^{J^{0}} c g\left(\theta_{j}\right) \cos n \theta_{j}, \\
& b \equiv\left\{\begin{array}{l}
1 \text { for } n=0, J^{0} \\
2 \text { for } 0<n<J^{0}
\end{array}, \quad c \equiv\left\{\begin{array}{l}
1 / 2 \text { for } j=0, J^{0} \\
1 \quad \text { for } 0<j<J^{0},
\end{array}\right.\right. \\
& g\left(\theta_{j}\right)=\sum_{n=0}^{J^{0}} g_{n} \cos n \theta_{j} .
\end{aligned}
$$

When using Grid [1], forward and inverse discrete sine transforms are performed as

$$
\begin{aligned}
h_{n} & =\frac{2}{J^{0}} \sum_{j=1}^{J^{0}-1} h\left(\theta_{j}\right) \sin n \theta_{j}, \\
h\left(\theta_{j}\right) & =\sum_{n=1}^{J^{0}-1} h_{n} \sin n \theta_{j}, \quad h\left(\theta_{0}\right)=h\left(\theta_{J^{0}}\right)=0 .
\end{aligned}
$$

Grid [-1] is the same as Grid [1], except that there are no grid points at the North and South poles. The zonal wavenumber components of scalar variables at the poles are zero except for $m=0$ (See Eq. (8)), and those of vector variables at the poles 
are zero except for $m=1$ (See Eqs. (47) and (48)). When we use Grid [-1] and the values at the poles are known to be zero, forward and inverse discrete cosine transforms can be performed using Eq. (68) and forward and inverse discrete sine transforms can be performed using Eq. (69) in the same way as for Grid [1]. When we use Grid [-1] and the values at the poles are unknown (i.e., the zonal wavenumber components of scalar variables for $m=0$, and those of vector variables for $5 m=1$ ), the inverse discrete cosine transform can be performed using Eq. (68b), but the forward discrete cosine transform cannot be performed using Eq. (68a). We can calculate the expansion coefficients $g_{n}$ from $g\left(\theta_{j}\right)$ in the following way. Eq. (68b) is multiplied by $\sin \theta_{j}$, and we define $\hat{g}\left(\theta_{j}\right)$ as

$$
\hat{g}\left(\theta_{j}\right) \equiv g\left(\theta_{j}\right) \sin \theta_{j}=\sum_{n=0}^{J^{0}} g_{n} \sin \theta_{j} \cos n \theta_{j} .
$$

Since the values $\hat{g}\left(\theta_{j}\right)$ at the poles $\left(j=0, J^{0}\right)$ are zero, we can expand $\hat{g}\left(\theta_{j}\right)$ as

$$
\hat{g}\left(\theta_{j}\right)=\sum_{n=1}^{J^{0}-1} \hat{g}_{n} \sin n \theta_{j} .
$$

The expansion coefficients $\hat{g}_{n}$ can be obtained from $\hat{g}\left(\theta_{j}\right)$ in the same way as in Eq. (69a) by forward discrete sine transform:

$$
\hat{g}_{n}=\frac{2}{J^{0}} \sum_{j=1}^{J^{0}-1} \hat{g}\left(\theta_{j}\right) \sin n \theta_{j} .
$$

From Eqs. (70) and (71), we obtain

$$
\sum_{n=0}^{J^{0}-2} g_{n} \sin \theta \cos n \theta=\sum_{n=1}^{J^{0}-1} \hat{g}_{n} \sin n \theta,
$$

$$
g_{J^{0}-1}=g_{J^{0}}=0 \text {, }
$$

By using Eq. (A2a), we obtain

$$
\sum_{n=0}^{J^{0}-2} g_{n} \sin \theta \cos n \theta=\left(g_{0}-\frac{g_{2}}{2}\right) \sin \theta+\sum_{n=1}^{J^{0}-3}\left(\frac{g_{n-1}}{2}-\frac{g_{n+1}}{2}\right) \sin n \theta+\frac{g_{J^{0}-3}}{2} \sin \left(J^{0}-2\right) \theta+\frac{g_{J^{0}-2}}{2} \sin \left(J^{0}-1\right) \theta .
$$

By substituting Eq. (74) into Eq. (73a) and comparing the left and right sides of the equation, we obtain

$$
\hat{g}_{n}=\left\{\begin{array}{cl}
g_{0}-\frac{g_{2}}{2} & \text { for } n=1 \\
\frac{g_{n-1}}{2}-\frac{g_{n+1}}{2} & \text { for } n=2, \ldots, J^{0}-3 \\
\frac{g_{J^{0}-3}}{2} & \text { for } n=J^{0}-2 \\
\frac{g_{J^{0}-2}}{2} & \text { for } n=J^{0}-2
\end{array}\right.
$$

We can calculate $\hat{g}\left(\theta_{j}\right)$ from $g\left(\theta_{i}\right)$ using Eq. (70), calculate $\hat{g}_{n}$ from $\hat{g}\left(\theta_{j}\right)$ using Eq. (72), and calculate $g_{n}$ from $\hat{g}_{n}$ using Eq. (75). 


\subsection{Zonal Fourier filter}

In regular longitude-latitude grids, the longitudinal grid spacing becomes narrow at high latitudes. In DFS methods, the zonal Fourier filter (Merilees 1974; Boer and Steinberg 1975; Cheong 2000a), which filters out the high zonal wavenumber components at high latitudes, is usually used to obtain a more uniform resolution. In this study, we set the largest zonal

5 wavenumber $M_{\mathrm{f}}$ at each latitude as

$$
M_{\mathrm{f}}\left(\theta_{j}\right)=\min \left(M, M_{0}+M \sin \left(\theta_{j}\right)\right),
$$

where we use the value $M_{0}=20$ to make the resolution similar to that in the reduced grid of Miyamoto (2006). The values of $T_{m}^{\mathrm{c}}\left(\theta_{j}\right)$ and $T_{m}^{\mathrm{s}}\left(\theta_{j}\right)$ in Eq. (7) are set to zero for $m>M_{\mathrm{f}}\left(\theta_{j}\right)$ during the spectral transform. The use of a reduced grid (Hortal and Simmons, 1991; Juang, 2004; Miyamoto, 2006) has a similar effect to the zonal Fourier filter.

\subsection{Laplacian operator and Poisson equation}

The calculation of the Laplacian operator and the Poisson equation in the new DFS method is described in this section. In the equation

$$
g(\lambda, \theta)=\nabla^{2} f(\lambda, \theta)=\frac{1}{a^{2}}\left[\frac{1}{\sin ^{2} \theta} \frac{\partial^{2} f}{\partial \lambda^{2}}+\frac{1}{\sin \theta} \frac{\partial}{\partial \theta}\left(\sin \theta \frac{\partial f}{\partial \theta}\right)\right],
$$

where $\nabla^{2}$ is the Laplacian operator, the variables $f$ and $g$ are expanded zonally using Eq. (7) as

$$
\begin{aligned}
& f(\lambda, \theta) \cong \sum_{m=0}^{M} f_{m}^{\mathrm{c}}(\theta) \cos m \lambda+\sum_{m=1}^{M} f_{m}^{\mathrm{s}}(\theta) \sin m \lambda \\
& g(\lambda, \theta) \cong \sum_{m=0}^{M} g_{m}^{\mathrm{c}}(\theta) \cos m \lambda+\sum_{m=1}^{M} g_{m}^{\mathrm{s}}(\theta) \sin m \lambda .
\end{aligned}
$$

The variables $f_{m}^{\mathrm{c}}(\theta), f_{m}^{\mathrm{s}}(\theta), g_{m}^{\mathrm{c}}(\theta)$, and $g_{m}^{\mathrm{s}}(\theta)$ are expanded meridionally using Eq. (8) as

$$
f_{m}^{c}(\theta) \cong f_{m}^{c, N}(\theta) \equiv \begin{cases}\sum_{n=0}^{N} f_{n, m}^{c} \cos n \theta, & \text { for } m=0, \\ \sum_{n=0}^{N-1} f_{n, m}^{c} \sin \theta \cos n \theta, & \text { for } m=1, \\ \sum_{n=1}^{N-1} f_{n, m}^{c} \sin \theta \sin n \theta, & \text { for even } m \geq 2, \\ \sum_{n=1}^{N-2} f_{n, m}^{c} \sin ^{2} \theta \sin n \theta, & \text { for odd } m \geq 3,\end{cases}
$$




$$
g_{m}^{\mathrm{c}}(\theta) \cong g_{m}^{\mathrm{c}, N}(\theta) \equiv \begin{cases}\sum_{n=0}^{N} g_{n, m}^{\mathrm{c}} \cos n \theta, & \text { for } m=0, \\ \sum_{n=0}^{N-1} g_{n, m}^{\mathrm{c}} \sin \theta \cos n \theta, & \text { for } m=1, \\ \sum_{n=1}^{N-1} g_{n, m}^{\mathrm{c}} \sin \theta \sin n \theta, & \text { for even } m \geq 2, \\ \sum_{n=1}^{N-2} g_{n, m}^{\mathrm{c}} \sin ^{2} \theta \sin n \theta, & \text { for odd } m \geq 3 .\end{cases}
$$

The equations for $f_{m}^{\mathrm{s}}(\theta)$ and $g_{m}^{\mathrm{s}}(\theta)$ for $m \geq 1$ are the same as Eqs. (80) and (81), respectively, except that the subscript $\mathrm{c}$ is replaced with s. We define the truncated variables $f^{N, M}(\theta)$ and $g^{N, M}(\theta)$ as

$$
\begin{aligned}
& f^{N, M}(\lambda, \theta) \equiv \sum_{m=0}^{M} f_{m}^{\mathrm{c}, N}(\theta) \cos m \lambda+\sum_{m=1}^{M} f_{m}^{\mathrm{s}, N}(\theta) \sin m \lambda, \\
& g^{N, M}(\lambda, \theta) \equiv \sum_{m=0}^{M} g_{m}^{\mathrm{c}, N}(\theta) \cos m \lambda+\sum_{m=1}^{M} g_{m}^{\mathrm{s}, N}(\theta) \sin m \lambda .
\end{aligned}
$$

From Eq. (82a), we obtain

$$
\begin{aligned}
\nabla^{2} f^{N, M}(\lambda, \theta)= & \sum_{m=0}^{M} \frac{1}{a^{2}}\left[\frac{-m^{2}}{\sin ^{2} \theta} f_{m}^{c, N}(\theta)+\frac{1}{\sin \theta} \frac{\partial}{\partial \theta}\left(\sin \theta \frac{\partial f_{m}^{c, N}(\theta)}{\partial \theta}\right)\right] \cos m \lambda \\
& +\sum_{m=1}^{M} \frac{1}{a^{2}}\left[\frac{-m^{2}}{\sin ^{2} \theta} f_{m}^{s, N}(\theta)+\frac{1}{\sin \theta} \frac{\partial}{\partial \theta}\left(\sin \theta \frac{\partial f_{m}^{s, N}(\theta)}{\partial \theta}\right)\right] \sin m \lambda .
\end{aligned}
$$

Here we use the Galerkin method to calculate the Laplacian operator and the Poisson equation, and obtain

$$
\begin{aligned}
& \frac{1}{2 \pi^{2}} \int_{0}^{2 \pi} \int_{0}^{\pi} S_{n, m}(\theta) \cos m \lambda R^{g}(\lambda, \theta) d \theta d \lambda=0, \\
& \frac{1}{2 \pi^{2}} \int_{0}^{2 \pi} \int_{0}^{\pi} S_{n, m}(\theta) \sin m \lambda R^{g}(\lambda, \theta) d \theta d \lambda=0,
\end{aligned}
$$

where the residual

$$
R^{g}(\lambda, \theta) \equiv g^{N, M}(\lambda, \theta)-\nabla^{2} f^{N, M}(\lambda, \theta)
$$

is orthogonal to each of the new DFS basis functions $S_{m, n}(\theta) \cos m \lambda$ and $S_{m, n}(\theta) \sin m \lambda$ (see Sect. 2.4).

15 We can also use the least-squares method described in Sect. 2.3 instead of the Galerkin method so that the following error $H$ (the squared $\mathrm{L}_{2}$ norm of the residual) is minimized:

$$
H \equiv \frac{1}{2 \pi^{2}} \int_{0}^{2 \pi} \int_{0}^{\pi} R^{g}(\lambda, \theta)^{2} d \theta d \lambda .
$$


When calculating $g$ by applying the Laplacian operator to a given $f, g_{n, m}^{\mathrm{c}}$ and $g_{n, m}^{\mathrm{s}}$ can be calculated from $\partial H / \partial g_{n, m}^{\mathrm{c}}$ and $\partial H / \partial g_{n, m}^{\mathrm{s}}$ using the least-squares method. The equations $\partial H / \partial g_{n, m}^{\mathrm{c}}$ and $\partial H / \partial g_{n, m}^{\mathrm{s}}$ give the equivalent equations to Eq. (84). When calculating $f$ from a given $g$ in the Poisson equation, $f_{n, m}^{c}$ and $f_{n, m}^{\mathrm{s}}$ can be calculated from $\partial H / \partial f_{n, m}^{\mathrm{c}}$ and $\partial H / \partial f_{n, m}^{\mathrm{s}}$ using the least-squares method. However, the equations derived from $\partial H / \partial f_{n, m}^{c}$ and $\partial H / \partial f_{n, m}^{s}$ are different from Eq. (84). If we use different equations for calculating $g$ from $f$ and $f$ from $g$, the original values are changed when calculating $g$ from $f$ followed by calculating $f$ from $g$, which is not good for numerical stability. Therefore, we use Eq. (84) for calculating both $g$ from $f$ and $f$ from $g$.

From Eqs. (82) to (85) and Eq. (A3) we derive

$$
\begin{aligned}
& \int_{0}^{\pi} S_{n, m}(\theta)\left\{g_{m}^{\mathrm{c}, N}(\theta)-\frac{1}{a^{2}}\left[\frac{-m^{2}}{\sin ^{2} \theta} f_{m}^{\mathrm{c}, N}(\theta)+\frac{1}{\sin \theta} \frac{\partial}{\partial \theta}\left(\sin \theta \frac{\partial f_{m}^{\mathrm{c}, N}(\theta)}{\partial \theta}\right)\right]\right\} d \theta=0, \\
& \int_{0}^{\pi} S_{n, m}(\theta)\left\{g_{m}^{\mathrm{s}, N}(\theta)-\frac{1}{a^{2}}\left[\frac{-m^{2}}{\sin ^{2} \theta} f_{m}^{\mathrm{s}, N}(\theta)+\frac{1}{\sin \theta} \frac{\partial}{\partial \theta}\left(\sin \theta \frac{\partial f_{m}^{\mathrm{s}, N}(\theta)}{\partial \theta}\right)\right]\right\} d \theta=0 .
\end{aligned}
$$

For $m=0$, we calculate $g_{n, m}^{\mathrm{c}}$ by using

$$
g_{m}^{\mathrm{c}, N}(\theta)=\frac{1}{a^{2}}\left[\frac{-m^{2}}{\sin ^{2} \theta} f_{m}^{\mathrm{c}, N}(\theta)+\frac{1}{\sin \theta} \frac{\partial}{\partial \theta}\left(\sin \theta \frac{\partial f_{m}^{\mathrm{c}, N}(\theta)}{\partial \theta}\right)\right],
$$

instead of Eq. (87) following Yee (1981) and Cheong (2000a) for ease of calculation. For $0 \leq m \leq 3$, the exact solutions of $g_{n, m}^{\mathrm{c}}$ can be obtained from Eq. (88) because the new DFS meridional basis functions for $0 \leq m \leq 3$ are the linear combination of the associated Legendre functions for $0 \leq m \leq 3$ and vice versa as described in Sect. 2.5.

For $m=0$, by substituting Eqs. (80) and (81) into Eq. (88) multiplied by $\sin ^{2} \theta$, transforming using Eq. (A5), and comparing both sides of the equation, we obtain

$$
-g_{n-2, m}^{\mathrm{c}}+2 g_{n, m}^{\mathrm{c}}-g_{n+2, m}^{\mathrm{c}}=\frac{1}{a^{2}}\left[(n-1)(n-2) f_{n-2, m}^{\mathrm{c}}-2 n^{2} f_{n, m}^{\mathrm{c}}+(n+1)(n+2) f_{n+2, m}^{\mathrm{c}}\right](0 \leq n \leq N),
$$

except for the following underlined values:

$$
\begin{array}{cc}
\underline{1} g_{1, m}^{\mathrm{c}}-g_{3, m}^{\mathrm{c}}=\cdots & (n=1), \\
-\underline{2} g_{0, m}^{\mathrm{c}}+2 g_{2, m}^{\mathrm{c}}-g_{4, m}^{\mathrm{c}}=\cdots & (n=2) .
\end{array}
$$

For $m=1$, by substituting Eqs. (80) and (81) into Eq. (87a) and using Eqs. (A2), (A4) and (A5), we obtain

$$
-g_{n-2, m}^{\mathrm{c}}+2 g_{n, m}^{\mathrm{c}}-g_{n+2, m}^{\mathrm{c}}=\frac{1}{a^{2}}\left[(n-1) n f_{n-2, m}^{\mathrm{c}}-\left(2 n^{2}+4 m^{2}\right) f_{n, m}^{\mathrm{c}}+(n+1) n f_{n+2, m}^{\mathrm{c}}\right] \quad(0 \leq n \leq N-1)
$$

except for the following underlined values:

$$
\begin{aligned}
& \underline{1} g_{1, m}^{\mathrm{c}}-g_{3, m}^{\mathrm{c}}=\cdots \quad(n=1), \\
& -2 g_{0, m}^{\mathrm{c}}+2 g_{2, m}^{\mathrm{c}}-g_{4, m}^{\mathrm{c}}=\frac{1}{a^{2}}\left[4 f_{0, m}^{\mathrm{c}}+\cdots\right] \quad(n=2) .
\end{aligned}
$$

For even $m \geq 2$, by substituting Eqs. (80) and (81) into Eq. (87a) and using Eqs. (A4) and (A5), we obtain 


$$
-g_{n-2, m}^{\mathrm{c}}+2 g_{n, m}^{\mathrm{c}}-g_{n+2, m}^{\mathrm{c}}=\frac{1}{a^{2}}\left[(n-1) n f_{n-2, m}^{\mathrm{c}}-\left(2 n^{2}+4 m^{2}\right) f_{n, m}^{\mathrm{c}}+(n+1) n f_{n+2, m}^{\mathrm{c}}\right] \quad(1 \leq n \leq N-1)
$$
except for the following underlined values:

$$
\underline{3} g_{1, m}^{\mathrm{c}}-g_{3, m}^{\mathrm{c}}=\cdots \quad(n=1)
$$

with no exceptions.

5 For odd $m \geq 3$, by substituting Eqs. (80) and (81) into Eq. (87a) and using Eqs. (A2), (A4) and (A5), we obtain

$$
\begin{aligned}
& g_{n-4, m}^{\mathrm{c}}-4 g_{n-2, m}^{\mathrm{c}}+6 g_{n, m}^{\mathrm{c}}-4 g_{n+2, m}^{\mathrm{c}}+g_{n+4, m}^{\mathrm{c}} \\
=\frac{1}{a^{2}}[ & -(n-2)(n-1) f_{n-4, m}^{\mathrm{c}}+\left(4 n^{2}-6 n+4+4 m^{2}\right) f_{n-2, m}^{\mathrm{c}}-\left(6 n^{2}+4+8 m^{2}\right) f_{n, m}^{\mathrm{c}} \\
& \left.\quad+\left(4 n^{2}+6 n+4+4 m^{2}\right) f_{n+2, m}^{\mathrm{c}}-(n+2)(n+1) f_{n+4, m}^{\mathrm{c}}\right] \quad(1 \leq n \leq N)
\end{aligned}
$$

except for the following underlined values:

$$
\begin{array}{cc}
\underline{10} g_{1, m}^{\mathrm{c}}-\underline{5} g_{3, m}^{\mathrm{c}}+g_{5, m}^{\mathrm{c}}=\frac{1}{a^{2}}\left[-\left(\underline{12}+\underline{12} m^{2}\right) f_{1, m}^{\mathrm{c}}+\cdots\right] & (n=1), \\
\underline{5} g_{2, m}^{\mathrm{c}}-4 g_{4, m}^{\mathrm{c}}+g_{6, m}^{\mathrm{c}}=\cdots & (n=2), \\
-\underline{5} g_{1, m}^{\mathrm{c}}+6 g_{3, m}^{\mathrm{c}}-4 g_{5, m}^{\mathrm{c}}+g_{7, m}^{\mathrm{c}}=\frac{1}{a^{2}}\left[\left(\underline{24}+\underline{4} m^{2}\right) f_{1, m}^{\mathrm{c}}+\cdots\right] & (n=3) .
\end{array}
$$

From Eq. (89), we obtain the following two linear simultaneous equations with tri-diagonal or penta-diagonal matrices:

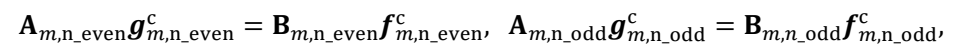

15 where $\boldsymbol{g}_{m, \text { even }}^{\mathrm{c}}$ and $\boldsymbol{g}_{m, \text { odd }}^{\mathrm{c}}$ are the vectors whose components are $g_{n, m}^{\mathrm{c}}\left(n\right.$ is even) and $g_{n, m}^{\mathrm{c}}(n$ is odd), respectively, and $\boldsymbol{f}_{m, n_{-} \text {even }}^{c}$ and $\boldsymbol{f}_{m, n_{-} \text {odd }}^{c}$ are the vectors whose components are $f_{n, m}^{c}\left(n\right.$ is even) and $f_{n, m}^{c}\left(n\right.$ is odd), respectively; $\mathbf{A}_{m, n_{-} \text {even }}$, $\mathbf{B}_{m, n_{-} \text {even }}, \mathbf{A}_{m, n_{-} \text {odd }}$, and $\mathbf{B}_{m, \text { n_odd }}$ are tri-diagonal or penta-diagonal matrices. $\boldsymbol{g}_{m, n \_e v e n}^{\mathbf{c}}$ and $\boldsymbol{g}_{m, n_{c} \text { odd }}^{\mathbf{c}}$ are calculated by

$$
\boldsymbol{g}_{m, \mathrm{n}_{\text {even }}}^{\mathrm{c}}=\mathbf{A}_{m, \mathrm{n}_{\text {even }}}^{-1} \mathbf{B}_{m, \mathrm{n}_{\text {even }}} \boldsymbol{f}_{m, \mathrm{n}_{\text {even }}}^{c}, \boldsymbol{g}_{m, \mathrm{n}_{\text {odd }}}^{\mathrm{c}}=\mathbf{A}_{m, \mathrm{n}_{\text {odd }}}^{-1} \mathbf{B}_{m, \mathrm{n}_{\text {odd }}} \boldsymbol{f}_{m, \mathrm{n}_{\text {odd }}}^{c}
$$

which can be solved efficiently as in Eq. (25).

By using Eq. (87b) instead of Eq. (87a), we obtain the equations to calculate $g_{n, m}^{\mathrm{s}}$ from $f_{n, m}^{\mathrm{s}}$, which are the same as Eqs. (89) to (91), except that the superscript $\mathrm{c}$ is replaced with the superscript $\mathrm{s}$.

We have verified that all the eigenvalues of the matrices $\mathbf{A}_{m, n_{-} \text {even }}^{-1} \mathbf{B}_{m, n_{-} \text {even }}$ and $\mathbf{A}_{m, n_{-} \text {odd }}^{-1} \mathbf{B}_{m, n_{-} \text {odd }}$ are negative real numbers for several truncation wavenumbers $M$ and $N$, but we have not yet proved that this is true for all truncation wavenumbers.

In the Poisson equation, $f$ is calculated from given $g$ in Eq. (77). We calculate $f$ from $g$ by the reverse calculation of $g$ from $f$ in Eq. (91). That is, we calculate $f$ from $g$ by

$$
\boldsymbol{f}_{m, \mathrm{n}_{-} \text {even }}^{\mathrm{c}}=\mathbf{B}_{m, \mathrm{n} \_ \text {even }}^{-1} \mathbf{A}_{m, \mathrm{n} \_ \text {even }} \boldsymbol{g}_{m, \mathrm{n}_{-} \text {even }}^{\mathrm{c}}, \boldsymbol{f}_{m, \mathrm{n} \_ \text {odd }}^{\mathrm{c}}=\mathbf{B}_{m, \mathrm{n}_{-} \text {odd }}^{-1} \mathbf{A}_{m, \mathrm{n} \_ \text {odd }} \boldsymbol{f}_{m, \mathrm{n}_{-} \text {odd }}^{\mathrm{c}},
$$

except when $m=0$ and $n$ is even. For $m=0, f_{n=0, m=0}^{c}$ disappears in Eq. (89a). The coefficients $f_{n, m=0}^{c}$ (even $n \geq 2$ ) are calculated from $g_{n, m=0}^{c}$ (even $n \geq 2$ ) by Eq. (89a). The value $f_{n=0, m=0}^{c}$ is calculated from $f_{n, m=0}^{c}($ even $n \geq 2)$ so that the 
global mean of $f$ is zero using Eq. (B1). $\boldsymbol{f}_{m, \mathrm{n}_{-} \text {even }}^{\mathrm{s}}$ and $\boldsymbol{f}_{m, \mathrm{n}_{-} \text {odd }}^{\mathrm{s}}$ are also calculated from the equations where the superscripts $\mathrm{c}$ in Eq. (92) are replaced with the superscript $\mathrm{s}$.

\subsection{The Helmholtz equation}

From Eq. (91), Eq. (77) is represented as

$$
g=\mathrm{A}^{-1} \mathrm{~B} f,
$$

where the subscripts $m, \mathrm{n}_{-}$even and $\mathrm{n}_{-}$odd, and the superscripts $\mathrm{c}$ and s are omitted. The matrix $\mathbf{A}^{\mathbf{- 1}} \mathbf{B}$ represents the Laplacian operator $\nabla^{2}$ in spectral space.

The Helmholtz equation is

$$
f-\varepsilon \nabla^{2} f=\left\{1-\varepsilon \frac{1}{a^{2}}\left[\frac{1}{\sin ^{2} \theta} \frac{\partial^{2}}{\partial \lambda^{2}}+\frac{1}{\sin \theta} \frac{\partial}{\partial \theta}\left(\sin \theta \frac{\partial}{\partial \theta}\right)\right]\right\} f=g,
$$

where $f$ is calculated from given $g$. Equation (94) is represented as

$$
\left(\mathbf{I}-\varepsilon \mathbf{A}^{-1} \mathbf{B}\right) \boldsymbol{f}=\boldsymbol{g} .
$$

From Eq. (95), $\boldsymbol{f}$ is calculated from $\boldsymbol{g}$ by

$$
\boldsymbol{f}=(\mathbf{A}-\varepsilon \mathbf{B})^{-\mathbf{1}} \mathbf{A} \boldsymbol{g} .
$$

Since $\mathbf{A}-\varepsilon \mathbf{B}$ is a penta-diagonal or tri-diagonal matrix, Eq. (96) can be efficiently solved as in Eq. (25). Similarly, the

is represented as

$$
f-\varepsilon_{1} \nabla^{2} f=\nabla^{2} g
$$

$$
\left(\mathbf{I}-\varepsilon_{1} \mathbf{A}^{-1} \mathbf{B}\right) \boldsymbol{f}=\mathbf{A}^{-1} \mathbf{B} \boldsymbol{g}
$$

From Eq. (98), $\boldsymbol{f}$ is calculated from $\boldsymbol{g}$ by

$$
f=\left(\mathbf{A}-\varepsilon_{1} \mathbf{B}\right)^{-1} \mathbf{B} \boldsymbol{g} .
$$

\subsection{Horizontal diffusion}

The horizontal diffusion is calculated in the same way as in Cheong et al. (2004). The equation for fourth-order hyperdiffusion is

$$
f+\varepsilon \nabla^{4} f=g,
$$

where $f$ is calculated from $g$. Equation (100) can be converted into

$$
\left(1+i \sqrt{\varepsilon} \nabla^{2}\right)\left(1-i \sqrt{\varepsilon} \nabla^{2}\right) f=g
$$

where $i=\sqrt{-1}$. Equation (101) is represented as

$$
\left(\mathbf{I}+i \sqrt{\varepsilon} \mathbf{A}^{-\mathbf{1}} \mathbf{B}\right)\left(\mathbf{I}-i \sqrt{\varepsilon} \mathbf{A}^{-\mathbf{1}} \mathbf{B}\right) \boldsymbol{f}=\boldsymbol{g},
$$

from which we obtain the equation to calculate $\boldsymbol{f}$ from $\boldsymbol{g}$ as

$$
\boldsymbol{f}=(\mathbf{A}-i \sqrt{\varepsilon} \mathbf{B})^{-\mathbf{1}} \mathbf{A}(\mathbf{A}+i \sqrt{\varepsilon} \mathbf{B})^{-\mathbf{1}} \mathbf{A} \boldsymbol{g} .
$$


Here, $\mathbf{A}-i \sqrt{\varepsilon} \mathbf{B}$ and $\mathbf{A}+i \sqrt{\varepsilon} \mathbf{B}$ are complex matrices and $\boldsymbol{f}$ and $\boldsymbol{g}$ are real column vectors. For efficient computation, two real column vectors can be combined into one complex column vector (Cheong et al., 2004); for example, $\boldsymbol{f}=\boldsymbol{f}^{\mathrm{c}}-i \boldsymbol{f}^{\mathrm{s}}$ and $\boldsymbol{g}=$ $\boldsymbol{g}^{\mathbf{c}}-i \boldsymbol{g}^{\mathrm{s}}$, where the superscript $\mathrm{c}$ indicates the zonal cosine component, and the superscript $\mathrm{s}$ indicates the zonal sine component.

\section{The error due to meridional wavenumber truncation in DFS expansion methods}

Here we examine the error due to the meridional wavenumber truncation when the same continuity conditions at the poles as Eq. (3) are satisfied. In the DFS method of Orszag (1974), only $f_{N-1, m}$ and $f_{N, m}$ are modified to satisfy Eq. (4) equivalent to Eq. (3). The DFS meridional basis functions of Cheong in Eq. (6) automatically satisfy the pole conditions in Eq. (3) for even $m$, but not for odd $m$. The new DFS meridional basis functions in Eq. (8) automatically satisfy the condition in Eq. (3)

10 for both even and odd $m$. We compare the error due to the wavenumber truncation among these DFS methods.

Figure 2 shows the error due to the wavenumber truncation when we use Grid [0] (see Sect. 2.9) with the number of latitudinal grid points $J=64$. The original values of $F_{m}\left(\theta_{j}\right)$ are set to one at grid points north of $30^{\circ} \mathrm{N}$, and zero at grid points south of $30^{\circ} \mathrm{N}$. The original values are meridionally transformed from grid space to spectral space (forward transform), truncated with $N=42$, and then transformed back from spectral space to grid space (inverse transform) to obtain the truncated

15 reconstruction of $F_{m}\left(\theta_{j}\right)$.

In the method of Orszag using Eq. (2), a very large error occurs especially for odd $|m|\left(\geq 3\right.$ ) (Fig. 2) when $f_{N-1, m}$ and $f_{N, m}$ are modified to satisfy the pole conditions in Eq. (4). Dividing $F_{m}\left(\theta_{j}\right)$ by $\sin \theta$ before the forward Fourier cosine transform for odd $|m|(\geq 3)$ also contributes to the large error.

In the method of Cheong using Eq. (6), large high wavenumber oscillations appear for even $m(\neq 0)$ in Fig. 2. Although

20 the basis functions in the method of Cheong for even $m(\neq 0)$ are the same as those in the new method, the expansion coefficients are calculated differently in the two methods. In the method of Cheong, the simple meridional truncation with $N<J$ after the forward Fourier sine transform of a variable divided by $\sin \theta$ causes the large high-wavenumber oscillations. The large oscillations appear when the original values abruptly change around the poles. In the case shown in Fig. 2, the original values near the North Pole are one, but the value at the North Pole abruptly becomes zero due to the pole conditions of Eq. (3). When $N=J$ for even $m(\neq 0)$, the forward transform followed by the inverse transform does not change the original values, and the oscillations do not appear. For this reason, Yoshimura and Matsumura (2005) and Yoshimura (2012) set $N=J$ for even $m$, to improve stability. The result in the method of Cheong for odd $|m|(\geq 3)$ is not shown in Fig. 2 because the method does not satisfy the condition of Eq. (3) for odd $m$.

In the new DFS method described in Sect. 2, the usual small oscillations from the Gibbs phenomenon appear in Fig. 2, but 
minimize the error. Because of this, the truncation with arbitrary $N<J$ does not cause large oscillations in the new DFS method.

Even when using the basis functions of Orszag in Eq. (2), we can obtain results equivalent to the new DFS method by calculating the expansion coefficients using the least-squares method with Lagrange multipliers to minimize the error while

5 satisfying the pole conditions in Eq. (4).

\section{Shallow water model on a sphere}

\subsection{Equations}

The prognostic equations of the shallow water model on a sphere are

$$
\begin{aligned}
& \frac{d \boldsymbol{v}}{d t}=-2(\boldsymbol{\Omega} \times \boldsymbol{v})_{\mathrm{H}}-g \nabla h, \\
& \frac{d\left(h-h_{\mathrm{s}}\right)}{d t}=-\left(h-h_{\mathrm{s}}\right) \nabla \cdot \boldsymbol{v},
\end{aligned}
$$

where $t$ is time, $\boldsymbol{v}$ is the horizontal wind vector, $h$ is the height, $h_{\mathrm{s}}$ is the surface height, $g$ is the acceleration due to gravity, $\boldsymbol{\Omega}$ is the 3-dimensional angular velocity of the earth's rotation, and the subscript $\mathrm{H}$ indicates the horizontal component. Equation (104) is transformed for the advective treatment of the Coriolis term (Temperton, 1997) into

$$
\frac{d(\boldsymbol{v}+2 \boldsymbol{\Omega} \times \boldsymbol{r})}{d t}=-g \nabla h,
$$

15 where $\boldsymbol{r}$ is the 3-dimensional position vector from the Earth's center. Equation (105) is transformed for the spatially averaged Eulerian treatment of mountains (Ritchie and Tanguay, 1996) into

$$
\frac{d h}{d t}=-\left(h-h_{\mathrm{s}}\right) \nabla \cdot \boldsymbol{v}+\boldsymbol{v} \cdot \nabla h_{\mathrm{s}}
$$

\subsection{Time integration method}

A two-time-level semi-implicit semi-Lagrangian scheme (e.g., Temperton et al., 2001) and the Stable Extrapolation TwoTime-Level Scheme (SETTLS; Hortal, 2002) are adopted to discretize Eqs. (106) and (107) in time as

$$
\begin{gathered}
\frac{(\boldsymbol{v}+2 \boldsymbol{\Omega} \times \boldsymbol{r})^{+}-(\boldsymbol{v}+2 \boldsymbol{\Omega} \times \boldsymbol{r})_{\mathrm{D}}^{0}}{\Delta t}=-\left\{\frac{g\left(\nabla h_{\mathrm{D}}^{(+)}+\nabla h^{0}\right)}{2}-\beta_{\mathbf{v}} \frac{g\left(\nabla h_{\mathrm{D}}^{(+)}+\nabla h^{0}\right)}{2}+\beta_{\mathbf{v}} \frac{g\left(\nabla h_{\mathrm{D}}^{0}+\nabla h^{+}\right)}{2}\right\} \\
\frac{h^{+}-h_{\mathrm{D}}^{0}}{\Delta t}=\left\{-\frac{\left[\left(h-h_{s}\right) D\right]_{\mathrm{D}}^{(+)}+\left[\left(h-h_{s}\right) D\right]^{0}}{2}+\frac{\left[\boldsymbol{v} \cdot \nabla h_{s}\right]_{\mathrm{D}}^{(+)}+\left[\boldsymbol{v} \cdot \nabla h_{\mathrm{s}}\right]^{0}}{2}\right. \\
\left.+\beta_{h} \frac{[\bar{h} D]_{\mathrm{D}}^{(+)}+[\bar{h} D]^{0}}{2}-\beta_{h} \frac{[\bar{h} D]_{\mathrm{D}}^{0}+[\bar{h} D]^{+}}{2}\right\}
\end{gathered}
$$

where 


$$
D \equiv \nabla \cdot v=\frac{1}{a}\left[\frac{1}{\cos \phi} \frac{\partial u}{\partial \lambda}+\frac{1}{\cos \phi} \frac{\partial v \cos \phi}{\partial \phi}\right]
$$

is horizontal divergence; $\Delta t$ is a timestep; the superscripts,- 0 , and + mean past time $(t-\Delta t)$, present time $(t)$, and future time $(t+\Delta t)$, respectively, and the superscript $(+)$ means future time $(t+\Delta t)$ extrapolated in time, for example, $h^{(+)}=$

$2 h^{0}-h^{-}$; the subscript D means the departure point, and the absence of the subscript D means the arrival point; $\bar{h}$ is a constant 5 value of height for semi-implicit linear terms; $\beta_{v}$ and $\beta_{h}$ are second-order decentering parameters (Yukimoto et al., 2011). Using $\beta_{v}$ and $\beta_{h}$ larger than 1.0 (e.g., 1.2) increases the effect of the semi-implicit scheme improving computational stability, but $\beta_{v}=\beta_{h}=1.0$ is used here because $\bar{h}$ larger than $h$ is enough for stable calculations in the shallow water model. The departure point $\boldsymbol{x}_{\mathrm{D}}$ is the upstream horizontal position from the arrival point $\boldsymbol{x}$ along the wind vector between present time $(t)$ and future time $(t+\Delta t)$. Here, the arrival point $\boldsymbol{x}$ is on a grid point, and the departure point $\boldsymbol{x}_{\mathrm{D}}$ is not generally on a grid point. Since the right-hand sides of Eqs. (108) and (109) are the time average between present time $(t)$ and future time $(t+\Delta t)$ and the spatial average between the departure point and the arrival point, these equations have second-order precision in time and space. In SETTLS, $\boldsymbol{x}_{\mathrm{D}}$ is calculated using

$$
x_{\mathrm{D}}=x-\frac{\boldsymbol{v}_{\mathrm{D}}^{(+)}+\boldsymbol{v}^{0}}{2} \Delta t
$$

However, when $\Delta t$ is longer than 30 minutes, using $\boldsymbol{v}_{\mathrm{D}}^{(+)}$extrapolated in time to calculate $\boldsymbol{x}_{\mathrm{D}}$ causes numerical instability in our experiments. To avoid instability when $\Delta t$ is 1 hour, here we use

$$
\begin{aligned}
& \boldsymbol{x}_{\mathrm{D}}=\boldsymbol{x}-\frac{\boldsymbol{v}_{\mathrm{D}}^{0}+\boldsymbol{v}^{\prime+}}{2} \Delta t, \\
& \boldsymbol{v}^{\prime+} \equiv \boldsymbol{v}_{\mathrm{D}}^{0}+(2 \boldsymbol{\Omega} \times \boldsymbol{r})_{\mathrm{D}}-2 \boldsymbol{\Omega} \times \boldsymbol{r}-\frac{g\left(\nabla h_{\mathrm{D}}^{(+)}+\nabla h^{0}\right)}{2} \Delta t,
\end{aligned}
$$

instead of Eq. (111), where $\boldsymbol{v}^{\prime}+$ is a provisional future value obtained by discretizing Eq. (106) in an explicit semiLagrangian scheme. From Eq. (112), we obtain

$$
\boldsymbol{x}_{\mathrm{D}}=\boldsymbol{x}-\Delta t\left[\left(\boldsymbol{v}^{0}+\boldsymbol{\Omega} \times \boldsymbol{r}-\frac{g \Delta t \nabla h^{(+)}}{4}\right)_{\mathrm{D}}-\boldsymbol{\Omega} \times \boldsymbol{r}-\frac{g \Delta t \nabla h^{0}}{4}\right] .
$$

This method using a provisional future value to calculate $\boldsymbol{x}_{\mathrm{D}}$ is similar to the method in Gospodinov et al., (2001). Since the value with the subscript D depends on $\boldsymbol{x}_{\mathrm{D}}, \boldsymbol{x}_{\mathrm{D}}$ is calculated iteratively from Eq. (113) (e.g., Ritchie, 1995; Temperton et al., 2001). Since $\boldsymbol{x}_{\mathrm{D}}$ is not generally on the grid point, the value at $\boldsymbol{x}_{\mathrm{D}}$ is calculated by spatial interpolation from nearby grid points. In the right-hand side of Eq. (113), the value at $\boldsymbol{x}_{\mathrm{D}}$ with the subscript $\mathrm{D}$ is calculated by third-order Lagrange interpolation.

Eqs. (108) and (109) are transformed into

$$
\begin{aligned}
& \boldsymbol{v}^{+}+\frac{\beta_{\mathbf{v}} \Delta t}{2} g \nabla h^{+}=\boldsymbol{R}_{\boldsymbol{v}}, \\
& \quad \boldsymbol{R}_{\boldsymbol{v}} \equiv\left[\mathbf{v}^{0}+2 \boldsymbol{\Omega} \times \boldsymbol{r}-\frac{\Delta t}{2} g\left(\nabla h^{(+)}-\beta_{\mathbf{v}} \nabla h^{(+)}+\beta_{\mathbf{v}} \nabla h^{0}\right)\right]_{\mathrm{D}}-2 \boldsymbol{\Omega} \times \boldsymbol{r}-\frac{\Delta t}{2} g\left(\nabla h^{0}-\beta_{\mathbf{v}} \nabla h^{0}\right),
\end{aligned}
$$




$$
\begin{aligned}
h^{+}+\frac{\beta_{h} \Delta t}{2} \bar{h} D^{+} & =R_{h}, \\
R_{h} \equiv h^{0}+\frac{\Delta t}{2}\left\{\left[\left(-\left(h-h_{s}\right) D+\boldsymbol{v}\right.\right.\right. & \left.\left.\left.\cdot \nabla h_{s}\right)\right]^{(+)}+\beta_{h} \bar{h} D^{(+)}-\beta_{h} \bar{h} D^{0}\right\}_{\mathrm{D}} \\
& +\frac{\Delta t}{2}\left\{\left[\left(-\left(h-h_{s}\right) D+\boldsymbol{v} \cdot \nabla h_{s}\right)\right]^{0}+\beta_{h} \bar{h} D^{0}\right\} .
\end{aligned}
$$

In Eqs. (114b) and (115b), the values at $\boldsymbol{x}_{\mathrm{D}}$ with the subscript $\mathrm{D}$ are calculated by fifth-order and third-order Lagrange results in our experiments. From Eq. (114), we obtain

$$
\begin{aligned}
D^{+}+\frac{\beta_{\mathbf{v}} \Delta t}{2} g \nabla^{2} h^{+} & =R_{D}, \\
\zeta^{+} & =R_{\zeta},
\end{aligned}
$$

where

$$
\zeta \equiv \boldsymbol{k} \cdot \nabla \times \boldsymbol{v}=\frac{1}{a}\left[\frac{1}{\cos \phi} \frac{\partial v}{\partial \lambda}-\frac{1}{\cos \phi} \frac{\partial u \cos \phi}{\partial \phi}\right]
$$

is vorticity, $\boldsymbol{k} \equiv \boldsymbol{r} /|\boldsymbol{r}|$ is the vertical unit vector, $R_{D} \equiv \nabla \cdot \boldsymbol{R}_{\boldsymbol{v}}$ and $R_{\zeta} \equiv \boldsymbol{k} \cdot \nabla \times \boldsymbol{R}_{\boldsymbol{v}}$.

We calculate $h^{+}$and $\boldsymbol{v}^{+}$using the spectral transform method and the Galerkin method with the new DFS method as follows.

1. The scalar variable $R_{h}$ is transformed from grid space to spectral space using Eqs. (19) to (25). The components of the vector variable $\boldsymbol{R}_{v}=\left(R_{u}, R_{v}\right)$ in grid space are transformed to $R_{\chi}$ and $R_{\psi}$ in spectral space using Eqs. (51) to (62), where $R_{\chi}$ and $R_{\psi}$ are the velocity potential and the stream function of $\boldsymbol{R}_{\boldsymbol{v}}$, respectively.

2. $R_{D}$ and $R_{\zeta}$ are calculated by

$$
\begin{aligned}
& R_{D}=\nabla^{2} R_{\chi}, \\
& R_{\zeta}=\nabla^{2} R_{\psi},
\end{aligned}
$$

using Eqs. (89) and (91). $\zeta^{+}$is obtained from $R_{\zeta}$ using Eq. (117).

20 3. Equations (115a) and (119) are substituted into Eq. (116) and we obtain

$$
D^{+}-\left(\frac{\Delta t}{2}\right)^{2} \beta_{\mathbf{v}} \beta_{h} g \bar{h} \nabla^{2} D^{+}=\nabla^{2}\left(R_{\chi}-\frac{\Delta t}{2} \beta_{\mathbf{v}} g R_{h}\right) .
$$

$D^{+}$is calculated by solving the Helmholtz-like equation Eq. (121) using Eqs. (97) and (99).

4. $h^{+}$is calculated from $D^{+}$and $R_{h}$ using Eq. (115).

5. $\chi^{+}$and $\psi^{+}$are calculated from $D^{+}$and $\zeta^{+}$by solving the Poisson equations

$$
\begin{aligned}
& \nabla^{2} \chi^{+}=D^{+}, \\
& \nabla^{2} \psi^{+}=\zeta^{+},
\end{aligned}
$$

using Eqs. (89) and (92).

6. $\mathbf{v}^{+}=\left(u^{+}, v^{+}\right)$is calculated from $\chi^{+}$and $\psi^{+}$using Eq. (49) for $u_{n, m}^{c}$ and the similar equations for $u_{n, m}^{s}, v_{n, m}^{c}$, and $v_{n, m}^{s}$. 
Figure 3 shows the time series of forecast errors of the height for a 5-day integration in the Williamson test case 2 with $\alpha=$ $\pi / 2-0.05$ in the models with around $128 \times 64$ grid points and truncation wavenumber $N=63$ (DFS) or $N=62$ (SH), using no horizontal diffusion. The $\mathrm{L}_{1}, \mathrm{~L}_{2}$, and $\mathrm{L}_{\infty}$ errors are almost the same among the new DFS models using Grids [0], [1] and $[-1]$, the old DFS model and the SH model.

\section{$5 \quad 5.3$ Williamson test case 5}

The Williamson test case 5 simulates zonal flow over an isolated mountain. Figure 4 shows the predicted height after a 15day integration in Williamson test case 5 with $h_{0}=5960 \mathrm{~m}$. The result of the high-resolution SH model with $1920 \times 960$ grid points is regarded as the reference solution. Horizontal diffusion is not used. The errors with respect to the reference solution are almost the same for the new DFS models, the old DFS model, and the SH model with around $128 \times 64$ grid points. Figure

105 shows the longitudinal distributions of meridional wind at the grid points near the South Pole after a 15-day integration in the old and new DFS models using Grid [0] with $128 \times 64$ and $1920 \times 960$ grid points. While the zonal wavenumber 1 component is dominant in the new DFS model with $128 \times 64$ grid points, high zonal wavenumber noise appears in the old DFS model with $128 \times 64$ grid points. This difference is because the new DFS expansion method with the least-squares method improves numerical stability. By using this new expansion method, the high zonal wavenumber noise does not appear even in

15 the model that does not use the new DFS basis functions in Eq. (7) but uses the same DFS basis functions as in Eq. (7) except that the basis function for odd $m \geq 3$ is $\sin \theta \cos n \theta$ instead of $\sin ^{2} \theta \sin n \theta$. The result of this model is almost the same as that of the new model (Figure not shown). In the old DFS model at high resolution with $1920 \times 960$ grid points, the high wavenumber noise is not seen in Fig. 5. The higher the resolution, the smaller the high wavenumber noise becomes. Figure 6 shows the kinetic energy spectra of the horizontal winds (Lambert, 1984) after a 15-day integration in Williamson test case 5. The kinetic energy spectra in the DFS models are calculated from the SH expansion coefficients, which are obtained by firstly calculating the Gaussian grid-point values from the DFS coefficients using Eq. (8) for the new DFS method and Eq. (6) for the old DFS method, and secondly calculating the SH expansion coefficients from the Gaussian grid-point values by using a forward Legendre transform. In the old DFS model with $128 \times 64$ grid points, the high wavenumber components are larger than in the other models, which is related to the high wavenumber noise near the South Pole in Fig. 5. In the old DFS model with $1920 \times 960$ grid points, the high wavenumber components are a little larger than in the other models, but the differences are slight.

Figure 7 shows the predicted height after a 15-day integration in Williamson test case 5, which is the same as Fig. 4 except for the truncation wavenumber $N$. In our semi-implicit semi-Lagrangian models, we usually use $N$ satisfying $N \cong J-1(J$ is the number of latitudinal grid points), which is called linear truncation. However, here $N$ is determined to satisfy $N \cong 2(J-1) / 3$

30 to eliminate aliasing errors with quadratic nonlinearity (Orszag, 1971), which is called quadratic truncation. When using the quadratic truncation $N=42$, the new DFS models with Grids [0], [1], and [-1] are stable without horizontal diffusion, but the old DFS model without strong high-order horizontal diffusion is unstable. The numerical instability in the old DFS model 
occurs because of the high-wavenumber oscillations due to the quadratic wavenumber truncation for even $m(\neq 0)$, as explained in Sect. 3. The results for the new DFS models are almost the same as for the SH model. Figure 8 shows the kinetic energy spectrum of the horizontal winds after a 15-day integration in Williamson test case 5, which is the same as Fig. 6 except for the truncation wavenumber $N$. At the resolution $N=42$ with $128 \times 64$ grid points, the high wavenumber components are 5 a little larger in the SH model than in the new DFS model. At the resolution $N=639$ with $1920 \times 960$ grid points, small oscillations appear in the high wavenumber region in the SH model, but not in the new DFS models. In the SH model, the wind components $u$ and $v$ divided by $\sin \theta$ are transformed from grid space to spectral space (Ritchie, 1988; Temperton, 1991), which seems to be the cause of the small oscillation in the high wavenumber region. Another way to transform $u$ and $v$ from grid space to spectral space in the SH model is to use the vector harmonic transform (see Sect. 2.8), which avoids dividing $u$

10 and $v$ by $\sin \theta$ and improves the stability of the model (Swarztrauber, 2004). This approach is similar to the expansion method for $u$ and $v$ using the least-squares method in the new DFS method described in Sects. 2.7 and 2.8, and probably solves the problem with the high wavenumber components in the SH model. Alternatively, using $D$ and $\zeta$ instead of $u$ and $v$ as prognostic variables may mitigate this problem.

\subsection{Galewsky test case}

The Galewsky test case simulates a barotropically unstable mid-latitude jet. Figure 9 shows the predicted vorticity after a 6day integration in the Galewsky test case for the models at $1.3 \mathrm{~km}$ resolution with $30720 \times 15360$ grid points and the quadratic truncation $N=10239$, without horizontal diffusion. The result in the new DFS model using Grid [0] is almost the same as in the SH model. The old DFS model is unstable for the same reason as that shown in Fig. 7. Figure 10 shows the kinetic energy spectrum of horizontal winds after a 6-day integration in the Galewsky test case. The results are almost the same for the DFS models using Grid [0], [1] and [-1], and the SH model, but small oscillations appear near the truncation wavenumber in the SH model. This is probably for the same reason as in Williamson test case 5 in Fig. 8.

\subsection{Elapsed time}

Figure 11 shows the elapsed time for the 15-day integration in the Williamson test case 5 in the SH model and the new DFS model using Grid [0] at $20 \mathrm{~km}$ resolution with $1920 \times 960$ grid points and $N=958$ (SH) or $N=959$ (DFS), and that for the 6 -day integration in the Galewsky test case at $1.3 \mathrm{~km}$ resolution with $30720 \times 15360$ grid points and $N=10239$. We use one node (with two Intel Xeon Gold 6248 CPUs with 20 cores per CPU) of the FUJITSU Server PRIMERGY CX2550 M5 in the MRI. OpenMP parallelization is used, but MPI parallelization is not used. The elapsed time in the SH model is larger than in the DFS model, although the Legendre transform in the SH model is highly optimized for Intel AVX512. The higher the resolution, the larger is the difference of the elapsed time between the models. This is because the Legendre transform used in the SH model requires $\mathrm{O}\left(\mathrm{N}^{3}\right)$ operations while the Fourier cosine and sine transforms used in the DFS model require only $\mathrm{O}\left(N^{2} \log N\right)$ operations. 


\section{Conclusions and perspectives}

We have developed the new DFS method to improve the numerical stability of the DFS model, which has the following two improvements:

1. A new expansion method with the least-squares method is used to calculate the expansion coefficients so that the error due

5 to the meridional wavenumber truncation is minimized. The method also avoids dividing by $\sin \theta$ before taking the forward Fourier cosine or sine transform.

2. New DFS basis functions that guarantee that not only scalar variables, but also vector variables and the gradient of scalar variables, are continuous at the poles.

The equations obtained with the least-squares method are equivalent to those obtained with the Galerkin method. We also use the Galerkin method to solve partial differential equations such as the Poisson equation and the shallow water equations.

To test the new DFS method, we conducted experiments for the Williamson test cases 2 and 5, and the Galewsky test case in semi-implicit semi-Lagrangian shallow water models using the new DFS method with the three types of equally spaced latitudinal grids with or without the poles. We compared the results of the new DFS models using the new DFS method with the old DFS model using the method of Yoshimura and Matsumura (2005), and with the SH model.

15 The high zonal wavenumber noise of the meridional wind appears near the poles in the old DFS model, but not in the new DFS models. This is because the new DFS expansion method with the least-squares method improves the model's stability. In the old DFS model, a truncation wavenumber $N$ lower than the number of latitudinal grid points $J$ for even $m \neq 0$ causes numerical instability. In the new DFS model, an arbitrary meridional wavenumber truncation $N<J$ can be used without the stability problem because the error due to meridional wavenumber truncation is small when using the new DFS expansion method with the least-squares method. This is one of the merits of the new DFS method because the quadratic truncation $(N \cong 2(J-1) / 3)$ or the cubic truncation $(N \cong(J-1) / 2)$ is usually used in the Eulerian model and is also becoming to be used in the semi-Lagrangian model instead of the linear truncation $(N \cong J-1)$ for stability and efficiency at high resolutions (Hotta and Ujiie, 2018; Dueben et al., 2020). We have also confirmed that in the new DFS model, stable integration is possible in all test cases shown here even without using the zonal Fourier filter unlike in the old DFS model. Thus, the numerical stability of the semi-implicit semi-Lagrangian model using the new DFS method is very good.

The results of the new DFS shallow water model are almost the same as the SH shallow water model. But in the SH model without horizontal diffusion, small oscillations appear in the high wavenumber region of the kinetic energy spectrum in some cases, unlike in the new DFS model. This seems to be because the wind components $u$ and $v$ divided by $\sin \theta$ are transformed from grid space to spectral space in the SH model. This problem with the SH model can probably be solved by using the vector harmonic transform, which is similar to the expansion method for $u$ and $v$ using the least-squares method in the new DFS model.

The elapsed time in the new DFS model is shorter than in the SH model especially at high resolution because the Fourier transform requires only $\mathrm{O}\left(N^{2} \log N\right)$ operations, and the Legendre transform in the $\mathrm{SH}$ model requires $\mathrm{O}\left(N^{3}\right)$ operations. 
We developed hydrostatic and nonhydrostatic global atmospheric models using the old DFS method (Yoshimura and Matsumura, 2005; Yoshimura, 2012) and conducted typhoon prediction experiments in the nonhydrostatic global atmospheric model using the old DFS method in the Global $7 \mathrm{~km}$ mesh nonhydrostatic Model Intercomparison Project for improving TYphoon forecast (TYMIP-G7; Nakano et al., 2017). We have already developed a nonhydrostatic (or hydrostatic)

5 atmospheric model using the new DFS method, which will be described in another paper after improving the nonhydrostatic dynamical core as needed.

Code availability. The source codes of the DFS and SH shallow water models are available in the Supplement to the article and are licensed under a Creative Commons Attribution-NonCommercial-ShareAlike 4.0 International (CC BY-NC-SA 4.0) license. These models utilize the Netlib BIHAR library and the ISPACK library. The Netlib BIHAR library is available at https://www.netlib.org/bihar/ and is also included in the Supplement. The ISPACK library is available at https://www.gfddennou.org/arch/ispack/ispack-3.0.1.tar.gz.

Data availability. The results of model experiments are available at https://climate.mri15 jma.go.jp/pre/Yoshimura_DFS_SW_Testcase_2021/.

\section{Appendix A: Trigonometric identities}

We list here the trigonometric identities used in transforming the expressions in this paper.

The following identities are satisfied:

$$
\begin{aligned}
\sin n \theta \cos n^{\prime} \theta & =\frac{1}{2}\left[\sin \left(n+n^{\prime}\right) \theta+\sin \left(n-n^{\prime}\right) \theta\right] \\
\cos n \theta \sin n^{\prime} \theta & =\frac{1}{2}\left[\sin \left(n+n^{\prime}\right) \theta-\sin \left(n-n^{\prime}\right) \theta\right] \\
\cos n \theta \cos n^{\prime} \theta & =\frac{1}{2}\left[\cos \left(n+n^{\prime}\right) \theta+\cos \left(n-n^{\prime}\right) \theta\right] \\
\sin n \theta \sin n^{\prime} \theta & =\frac{1}{2}\left[-\cos \left(n+n^{\prime}\right) \theta+\cos \left(n-n^{\prime}\right) \theta\right]
\end{aligned}
$$

From Eq. (A1), the following identities are derived:

$$
\begin{aligned}
\sin \theta \cos n \theta & =\frac{1}{2}[\sin (n+1) \theta-\sin (n-1) \theta] \\
\sin \theta \sin n \theta & =\frac{1}{2}[-\cos (n+1) \theta+\cos (n-1) \theta] \\
\sin ^{2} \theta \sin n \theta & =\frac{1}{4}[-\sin (n-2) \theta+2 \sin n \theta-\sin (n+2) \theta]
\end{aligned}
$$




$$
\sin ^{2} \theta \cos n \theta=\frac{1}{4}[-\cos (n-2) \theta+2 \cos n \theta-\cos (n+2) \theta]
$$

From Eq. (A1), the following orthogonal relations in longitude are derived:

$$
\begin{aligned}
& \int_{0}^{2 \pi} \cos m \lambda \cos m^{\prime} \lambda d \lambda= \begin{cases}2 \pi & \text { for } m=m^{\prime}=0 \\
\pi & \text { for } m=m^{\prime} \neq 0 \\
0 & \text { for } m \neq m^{\prime}\end{cases} \\
& \int_{0}^{2 \pi} \cos m \lambda \sin m^{\prime} \lambda d \lambda=0 \\
& \int_{0}^{2 \pi} \sin m \lambda \sin m^{\prime} \lambda d \lambda= \begin{cases}\pi & \text { for } m=m^{\prime} \neq 0 \\
0 & \text { for } m \neq m^{\prime}\end{cases}
\end{aligned}
$$

Similarly, from Eq. (A1), the following orthogonal relations in latitude are derived:

$$
\begin{aligned}
& \int_{0}^{\pi} \cos n \theta \cos n^{\prime} \theta d \theta= \begin{cases}\pi & \text { for } n=n^{\prime}=0 \\
\frac{1}{2} \pi & \text { for } n=n^{\prime} \neq 0 \\
0 & \text { for } n \neq n^{\prime}\end{cases} \\
& \int_{0}^{\pi} \sin n \theta \sin n^{\prime} \theta d \theta=\left\{\begin{array}{cl}
\frac{1}{2} \pi & \text { for } n=n^{\prime} \neq 0 \\
0 & \text { for } n \neq n^{\prime}
\end{array}\right.
\end{aligned}
$$

By using Eq. (A1), the following relations are derived:

10

$$
\begin{gathered}
\sin \theta \frac{\partial}{\partial \theta}\left(\sin ^{l} \theta \cos n \theta\right)=\frac{n+l}{2} \sin ^{l} \theta \cos (n+1) \theta-\frac{n-l}{2} \sin ^{l} \theta \cos (n-1) \theta \\
\sin \theta \frac{\partial}{\partial \theta}\left[\sin \theta \frac{\partial}{\partial \theta}\left(\sin ^{l} \theta \cos n \theta\right)\right]=\frac{(n+l)(n+l+1)}{4} \sin ^{l} \theta \cos (n+2) \theta \\
-\frac{2 n^{2}-2 l^{2}+2 l}{4} \sin ^{l} \theta \cos n \theta+\frac{(n-l)(n-l-1)}{4} \sin ^{l} \theta \cos (n-2) \theta \\
\sin \theta \frac{\partial}{\partial \theta}\left(\sin ^{l} \theta \sin n \theta\right)=\frac{n+l}{2} \sin ^{l} \theta \sin (n+1) \theta-\frac{n-l}{2} \sin ^{l} \theta \sin (n-1) \theta \\
\sin \theta \frac{\partial}{\partial \theta}\left[\sin \theta \frac{\partial}{\partial \theta}\left(\sin ^{l} \theta \sin n \theta\right)\right]=\frac{(n+l)(n+l+1)}{4} \sin ^{l} \theta \sin (n+2) \theta \\
-\frac{2 n^{2}-2 l^{2}+2 l}{4} \sin ^{l} \theta \sin n \theta+\frac{(n-l)(n-l-1)}{4} \sin ^{l} \theta \sin (n-2) \theta
\end{gathered}
$$

\section{Appendix B: Calculation of global mean and latitudinal area weight}

The global mean value of $T^{N, M}(\lambda, \theta)$ in Eq. (11) can be calculated in spectral space by

$$
G=\frac{1}{4 \pi} \int_{0}^{2 \pi} \int_{0}^{\pi}\left(\sum_{m=0}^{M} T_{m}^{\mathrm{c}, N}(\theta) \cos m \lambda+\sum_{m=1}^{M} T_{m}^{\mathrm{s}, N}(\theta) \sin m \lambda\right) \sin \theta d \theta d \lambda
$$




$$
=\frac{1}{2} \int_{0}^{\pi} \sum_{n=0}^{N} T_{n, m=0}^{\mathrm{c}} \cos n \theta \sin \theta d \theta=\sum_{\substack{n=0 \\ \text { when } n \text { is even }}}^{N} \frac{T_{n, m=0}^{\mathrm{c}}}{1-n^{2}} .
$$

5 2. From $T_{m=0}^{\mathrm{c}(j)}\left(\theta_{k}\right)$, the meridional expansion coefficients $T_{n, m=0}^{\mathrm{c}(j)}(0 \leq n \leq N)$ are calculated by forward discrete cosine transform described in Sect. 2.10.

3. The value of $G$ calculated from $T_{n, m=0}^{\mathrm{c}(j)}$ using Eq. (C1) is considered as the latitudinal area weight $w_{j}$ at latitude $\theta_{j}$.

The latitudinal area weight $w_{j}\left(\theta_{j}\right)$ is used, for example, to calculate the global mean in the grid space.

\section{Author Contributions.}

10 HY developed a new DFS method and a shallow water model using the method, conducted model experiments, analysed data, and wrote the paper.

\section{Competing Interests.}

The author declares that there is no conflict of interest.

\section{Acknowledgements.}

15 We are grateful to Keiichi Ishioka (Kyoto University), Tadashi Tsuyuki (MRI), Daisuke Hotta (MRI), Masashi Ujiie (JMA), and other members of the MRI and JMA model development team for their useful comments. This work was supported by the Integrated Research Program for Advancing Climate Models (TOUGOU) Grant Number JPMXD0717935561 from the Ministry of Education, Culture, Sports, Science and Technology (MEXT), Japan.

\section{References}

Boer, G. J. and Steinberg, L.: Fourier series on spheres, Atmosphere, 13:4, 180-191, doi:10.1080/00046973.1975.9648396, 1975.

Boyd, J. P.: The choice of spectral functions on a sphere for boundary and eigenvalue problems: A comparison of Chebyshev, Fourier and associated Legendre expansions, Mon. Weather Rev., 106, 1184-1191, doi:10.1175/15200493(1978)106<1184:TCOSFO >2.0.CO;2, 1978 . 
Cheong, H.-B.: Double Fourier series on a sphere: applications to elliptic and vorticity equations, J. Comput. Phys., 157, 327349, doi:10.1006/jcph.1999.6385, 2000a.

Cheong, H.-B.: Application of double Fourier series to shallow water equations on a sphere, J. Comput. Phys., 165, 261-287, doi:10.2151/jmsj.2004.1301, 2000b.

5 Cheong, H.-B.: A dynamical core with double Fourier series: Comparison with the spherical harmonics method, Mon. Weather Rev., 134, 1299-1315, doi:10.1175/MWR3121.1, 2006.

Cheong, H.-B., Kwon, I.-H., and Goo, T.-Y.: Further study on the high-order double-Fourier-series spectral filtering on a sphere, J. Comput. Phys. 193, 180-197, doi:10.1016/j.jcp.2003.07.029, 2004.

Cheong, H.-B., Kwon, I.-H., Goo, T.-Y., and M.-J. Lee: High-order harmonic spectral filter with the double Fourier series on a sphere, J. Comput. Phys. 177, 313-335, doi:10.1006/jcph.2002.6997, 2002.

Cooley, J. W., and Tukey, J. W.: An algorithm for the machine calculation of complex Fourier series. Math. Comput., 19, 297301, doi:10.2307/2003354, 1965.

Dueben, P. D., Wedi, N., Saarinen, S., and Zeman, C. Global simulations of the atmosphere at $1.45 \mathrm{~km}$ grid-spacing with the Integrated Forecasting System, J. Meteorol. Soc. Jpn, 98(3), 551-572, doi:10.2151/jmsj.2020-016, 2020.

Galewsky J., Scott R. K., and Polvani L. M.: An initial-value problem for testing numerical models of the global shallowwater equations. Tellus A, 56, 429-440, doi:10.3402/tellusa.v56i5.14436, 2004.

Gander, W. and Golub, G. H.: Cyclic reduction - History and applications. Proceedings of the Workshop on Scientific Computing, 10-12, 1997.

Gospodinov, I., Spiridonov, V., and Geleyn, J.-F.: Second-order accuracy of two-time-level semi-Lagrangian schemes. Q. J. Roy. Meteor. Soc., 127, 1017-1033, doi:10.1002/qj.49712757317, 2001.

Hortal, M.: The development and testing of a new two-time-level semi-Lagrangian scheme (SETTLS) in the ECMWF forecast model. Q. J. Roy. Meteor. Soc., 128, 1671-1687, doi:10.1002/qj.200212858314, 2002.

Hortal, M. and Simmons, A. J.: Use of reduced Gaussian grids in spectral models: Mon. Weather Rev., 119, 1057-1074, doi:10.1175/1520-0493(1991)119<1057:UORGGI>2.0.CO;2, 1991.

Hotta, D. and Ujiie, M.: A nestable, multigrid-friendly grid on a sphere for global spectral models based on Clenshaw-Curtis quadrature, Q. J. Roy. Meteor. Soc., 144, 1382-1397, DOI: 10.1002/qj.3282, 2018.

Ishioka, K.: A New Recurrence Formula for Efficient Computation, J. Meteorol. Soc. Jpn., 96, 241-249, doi:10.2151/jmsj.2018-019, 2018.

Japan Meteorological Agency (JMA): Outline of the operational numerical weather prediction at the Japan Meteorological Agency, Appendix to WMO technical progress report on the global data-processing and forecasting system and numerical weather prediction, 229 pp., available at: http://www.jma.go.jp/jma/jma-eng/jma-center/nwp/outline2019-nwp/index.htm, 2019.

Juang, H.-M. H., 2004: A reduced spectral transform for the NCEP seasonal forecast global spectral atmospheric model. Mon. Weather Rev., 132, doi:10.1175/1520-0493(2004)132<1019:ARSTFT>2.0.CO;2, 1019-1035. 
Koo M.-S. and Hong, S.-Y.: Double Fourier series dynamical core with hybrid sigma-pressure vertical coordinate, Tellus A, 65, 19851, doi:10.3402/tellusa.v65i0.19851, 2013.

Kwon, I.-H., Cheong H.-B., Joh M., Chung I.-U., Cho C.-H., and Lee W.-J.: Application of double-Fourier-series spectral method to a large size problem: Two dimensional simulations of the shear instability on the sphere. J. Meteor. Soc. Japan, 82, doi:1301-1314, 10.2151/jmsj.2004.1301, 2004.

Lambert, S. J.: A global available potential energy-kinetic energy budget in terms of the two-dimensional wavenumber for the FGGE year, Atmos. Ocean, 22:3, 265-282, doi:10.1080/07055900.1984.9649199, 1984.

Layton, A. T., and Spotz, W. F.: A semi-Lagrangian double Fourier method for the shallow water equations on the sphere. J. Comput. Phys., 189, 180-196, doi:10.1016/S0021-9991(03)00207-9, 2003.

Merilees, P. E.: An alternative scheme for the simulation of a series of spherical harmonics, J. Appl. Meteorol., 12 (1), 224 227, doi:10.1175/1520-0450(1973)012<0224:AASFTS >2.0.CO;2, 1973a.

Merilees, P. E.: The pseudo-spectral approximation applied to the shallow water equations on a sphere, Atmosphere 11:1, 13-20, doi:10.1080/00046973.1973.9648342, 1973b.

Merilees, P. E.: Numerical experiments with the pseudospectral method in spherical coordinates, Atmosphere, 12:3, 77-96, doi:10.1080/00046973.1974.9648374, 1974.

Miyamoto, K.: Introduction of the Reduced Gaussian Grid into the Operational Global NWP model at JMA. CAS/JSC WGNE Research Activities in Atmospheric and Ocean Modelling, 36, 6.9-6.10., 2006.

Nakano, M., Wada, A., Sawada, M., Yoshimura, H., Onishi R., Kawahara, S., Sasaki W., Nasuno, T., Yamaguchi, M., Iriguchi, T., Sugi, M., and Takeuchi, Y.: Global 7km mesh nonhydrostatic Model Intercomparison Project for improving TYphoon forecast (TYMIP-G7): experimental design and preliminary results, Geosci. Model Dev., 10, 1363-1381, doi:10.5194/gmd-10-1363-2017, 2017.

Orszag, S. A.: On the elimination of aliasing in finite-difference schemes by filtering high-wavenumber components. J. Atmos. Sci., 28, 1074-1074, doi:10.1175/1520-0469(1971)028<1074:OTEOAI $2.0 . C O ; 2,1971$.

Orszag, S. A.: Fourier series on spheres, Mon. Weather Rev. 102, 56-75, doi:10.1175/15200493(1974)102<0056:FSOS>2.0.CO;2, 1974.

Park H., Hong S.-Y., Cheong H.-B., and Koo M.-S.: A Double Fourier Series (DFS) Dynamical Core in a Global Atmospheric Model with Full Physics, Mon. Weather Rev., 141 (9), 3052-3061, doi:10.1175/MWR-D-12-00270.1, 2013.

Ritchie, H.: Application of the semi-Lagrangian method to a spectral model of the shallow water equations. Mon. Weather Rev., 116, 1587-1598, doi:10.1080/07055900.1997.9687360, 1988.

Ritchie, H. and Tanguay, M.: A comparison of spatially averaged Eulerian and semi-Lagrangian treatments of mountains. Mon. Weather Rev., 124 (1), 167-181, doi:10.1175/1520-0493(1996)124<0167:ACOSAE>2.0.CO;2, 1996.

Ritchie, H., Temperton, C., and Simmons, A.: Implementation of the semi-Lagrangian method in a high-resolution version of the ECMWF forecast model. Mon. Weather Rev., 123, 489-514, doi:10.1175/15200493(1995)123<0489:IOTSLM>2.0.CO;2, 1995 . 
Schaeffer, N.: Efficient spherical harmonic transforms aimed at pseudospectral numerical simulations. Geochem. Geophys. Geosy., 14, 751-758, doi:10.1002/ggge.20071, 2013.

Sneeuw, N. and Bun, R.: Global spherical harmonic computation by two-dimensional Fourier methods, J. Geodesy, 70, 224232, 1996.

5 Spotz, W. F., Taylor, M. A., and Swartztrauber, P. N.: Fast shallow-water equations solvers in latitude-longitude coordinates, J. Comput. Phys. 145, 432, doi:10.1006/jcph.1998.6026, 1998.

Suda, R.: Fast spherical harmonic transform routine FLTSS applied to the shallow water test set. Mon. Weather Rev., 133, 634-648, doi:10.1175/MWR-2871.1, 2005.

Swarztrauber, P. N.: Vectorizing the FFTs, in Parallel Computations (G. Rodrigue, ed.), Academic Press, 1982, 51-83.

10 Swarztrauber, P. N.: The vector harmonic transform method for solving partial differential equations in spherical geometry. Mon. Weather Rev. 121, 3415-3437, doi:10.1175/1520-0493(1993)121<3415:TVHTMF>2.0.CO;2, 1993.

Swarztrauber, P. N.: Shallow water flow on the sphere. Mon. Weather Rev., 132, 3010-3018, doi:10.1175/MWR2829.1, 2004.

Swarztrauber, P. N., and Spotz, W. F.: Generalized discrete spherical armonic Transforms, J. Comput. Phys., 159, 213-230, doi:10.1006/jcph.2000.6431, 2000.

Temperton, C.: On scalar and vector transform methods for global spectral models, Mon. Weather Rev., 119, 1303-1307, doi:10.1175/1520-0493-119-5-1303.1, 1991.

Temperton, C.: Treatment of the Coriolis terms in semi-Lagrangian spectral models. Atmos, Ocean, 35:sup1, 293-302, doi:10.1080/07055900.1997.9687353, 1997.

Temperton, C., Hortal, M., and Simmons, A.: A two-time-level semi-Lagrangian global spectral model, Q. J. Roy. Meteor. Soc., 127, 111-127, doi:10.1002/qj.49712757107, 2001.

Tygert, M., 2008: Fast algorithms for spherical harmonic expansions, II. J. Comput. Phys., 227, doi:10.1016/j.jcp.2007.12.019, 4260-4279.

Wedi, N. P., Hamrud, M., and Mozdzynski, G.: A fast spherical harmonics transform for global NWP and climate models. Mon. Weather Rev. 141, 3450-3461, doi:10.1175/MWR-D-13-00016.1, 2013.

Williamson, D. L., Drake, J. B., Hack, J. J., Jacob, R., and Swarztrauber, P. N.: A standard test set for numerical approximations to the shallow water equations in spherical geometry, J. Comput. Phys., 102, 211-224, doi:10.1016/S00219991(05)80016-6, 1992.

Yee, S. Y. K.: Solution of Poisson's equation on a sphere by truncated double Fourier series, Mon. Weather Rev. 109, 501, doi:10.1175/1520-0493(1981)109<0501:SOPEOA >2.0.CO;2, 1981.

30 Yoshimura, H.: Development of a nonhydrostatic global spectral atmospheric model using double Fourier series, CAS/JSC WGNE Research Activities in Atmospheric and Ocean Modeling, 42, 3.05-3.06, 2012.

Yoshimura, H. and Matsumura, T.: A two-time-level vertically conservative semi-Lagrangian semi-implicit double Fourier series AGCM, CAS/JSC WGNE Research Activities in Atmospheric and Ocean Modeling, 35, 3.25-3.26, 2005. 
https://doi.org/10.5194/gmd-2021-168

Preprint. Discussion started: 8 July 2021

(c) Author(s) 2021. CC BY 4.0 License.

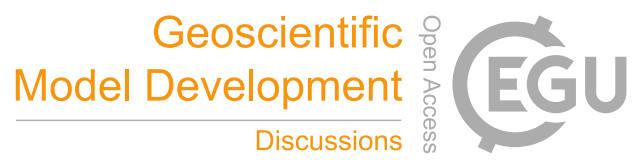

Yukimoto, S. and Coauthors: Meteorological Research Institute-Earth System Model Version 1 (MRI-ESM1) - Model Description - Technical Reports of the Meteorological Research Institute, No. 64, 2011.

Yukimoto, S. and Coauthors: The Meteorological Research Institute Earth System Model Version 2.0, MRI-ESM2.0: Description and Basic Evaluation of the Physical Component, J. Meteorol. Soc. Jpn., 97, 931-965, doi:10.2151/jmsj.2019-051, 2019. 
https://doi.org/10.5194/gmd-2021-168

Preprint. Discussion started: 8 July 2021

(c) Author(s) 2021. CC BY 4.0 License.

(c) (i)

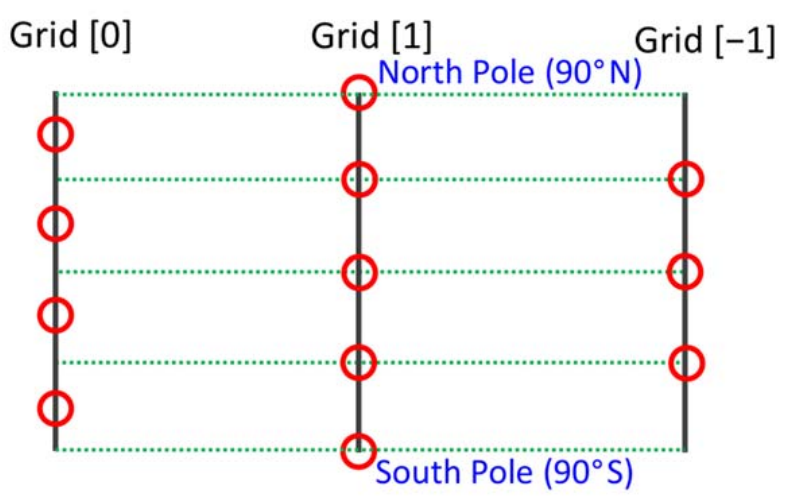

Figure 1. Grid [0], Grid[1], and Grid [-1] are three ways of arranging equally spaced latitudinal grid points when the grid interval $\Delta \theta=\pi / 4$. Red circles show the positions of the grid points.

5 
(a) $J=64 \quad N=42$ even $m \geq 2$

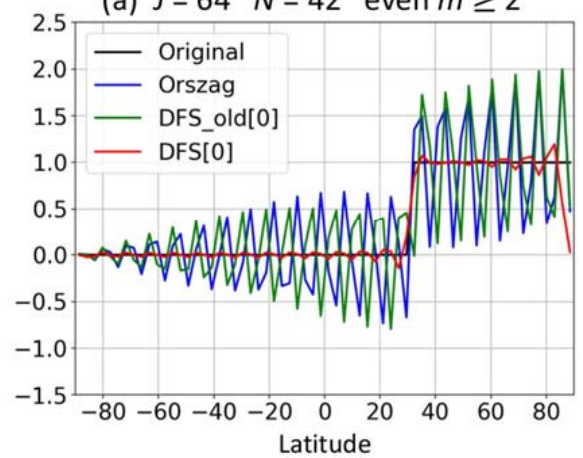

(b) $J=64 \quad N=42$ even $m \geq 3$

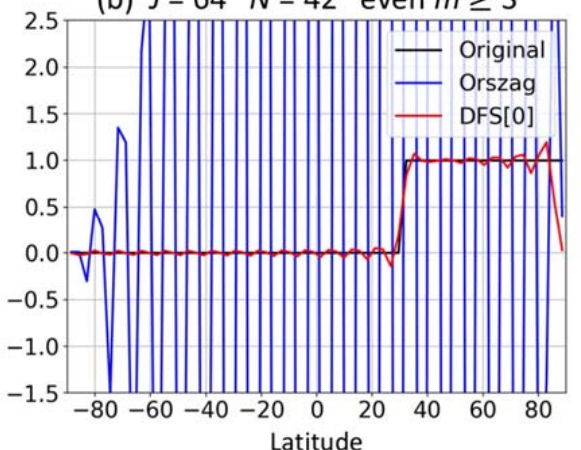

Figure 2. Change in values due to the meridional wavenumber truncation for (a) even $|m| \geq 2$, and (b) odd $|m| \geq 3$. We use Grid [0] with the number of latitudinal grid points $J=64$. Original values (black) are meridionally transformed from grid 5 space to spectral space, truncated with $N=42$, and transformed back from spectral space to grid space. Blue: Orszag's expansion method. Green: Cheong's expansion method. Red: the new expansion method. 


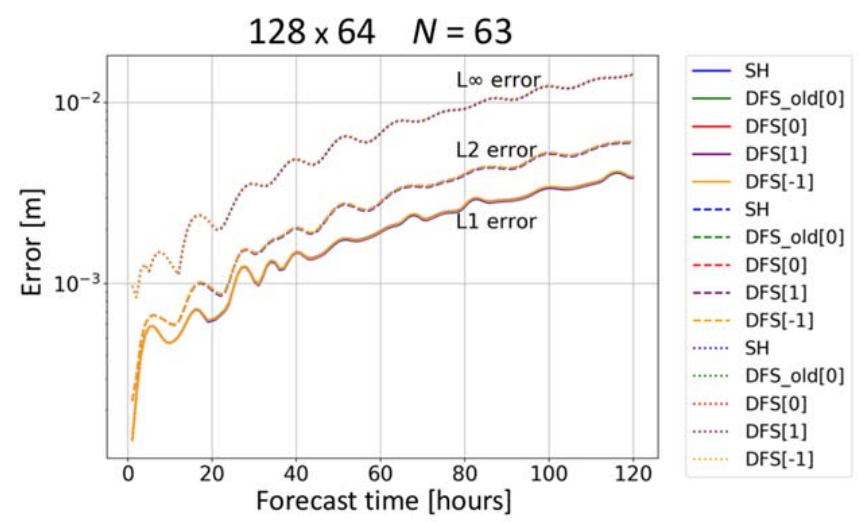

Figure 3. Time series of prediction error of height $(\mathrm{m})$ for 5 days (120 hours) integration in Williamson test case 2 $(\alpha=\pi / 2-0.05) .128 \times 64$ indicates the numbers of longitudinal and latitudinal grid points. $N$ is the truncation wavenumber.

5 Solid, dashed, and dotted lines represent $\mathrm{L}_{1}, \mathrm{~L}_{2}$, and $\mathrm{L}_{\infty}$ errors, respectively. The colors blue, green, red, purple, and orange represent the models using SH, old DFS with Grid [0], new DFS with Grid [0], new DFS with Grid [1], and new DFS with Grid [-1], respectively. 
(a) DFS [0] $128 \times 64 \quad N=63$

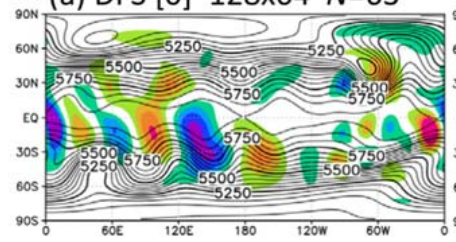

(d) DFS_old [0] $128 \times 64 \quad N=63$

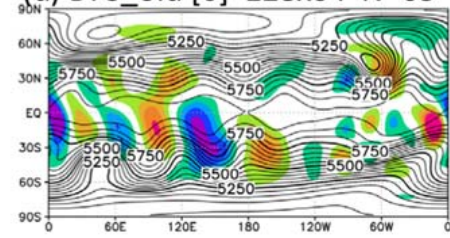

(b) DFS [1] $128 \times 65 \quad N=63$

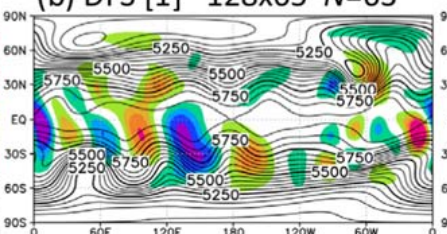

(c) DFS [-1] $128 \times 63 \quad N=63$

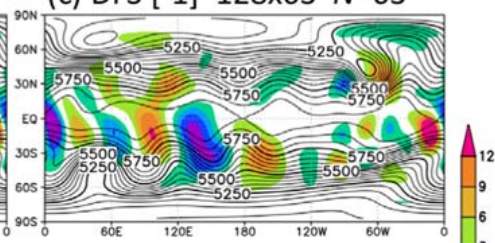

(e) $\mathrm{SH} 128 \times 64 \quad N=62$

(f) $\mathrm{SH} 1920 \times 960 \quad N=958$

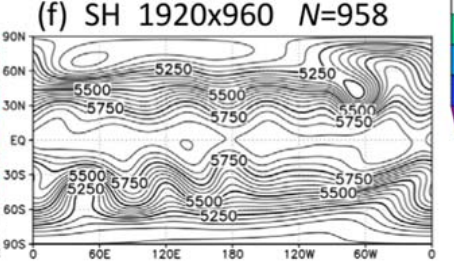

Figure 4. Predicted height (m) after a 15-day integration in Williamson test case 5. (a) New DFS model with Grid [0]. (b) New DFS model with Grid [1]. (c) New DFS model with Grid [-1]. (d) Old DFS model with Grid [0]. (e) SH model. (f) SH 5 model at high resolution, which is regarded as the reference solution. The number of longitudinal $(I)$ and latitudinal $(J)$ grid points is shown in the form $I \times J$. $N$ is the truncation wavenumber. Color shading shows the error with respect to the reference solution. 

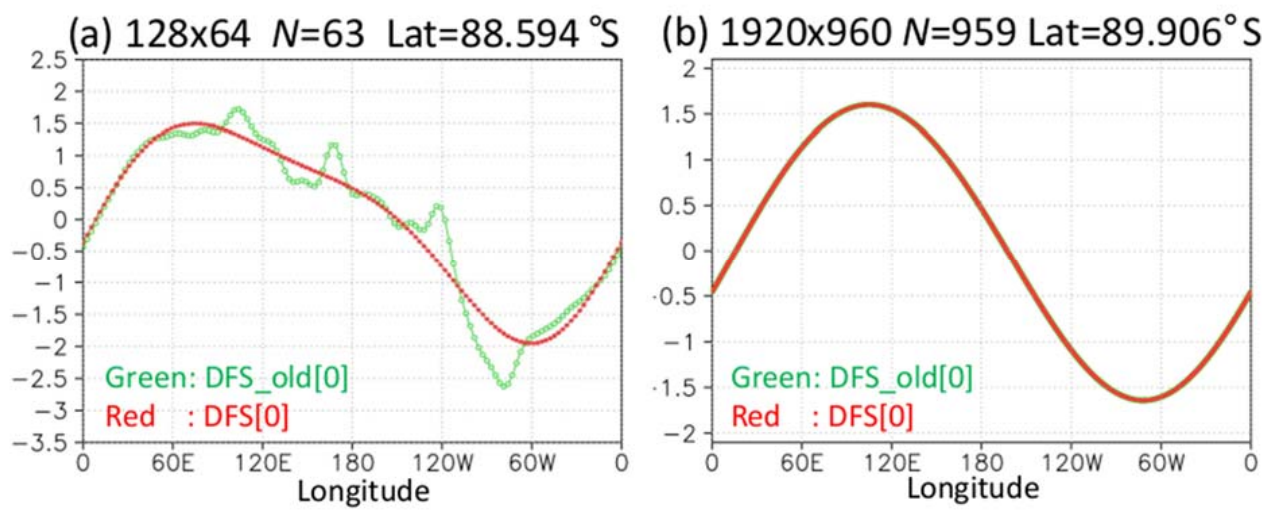

Figure 5. Longitudinal distributions of meridional wind $\left(\mathrm{m} \mathrm{s}^{-1}\right)$ at the grid points near the South Pole after a 15-day integration in Williamson test case 5. Results of the models using Grid [0] with (a) $128 \times 64$ grid points and truncation wavenumber $N=63$, and (b) $1920 \times 960$ grid points and $N=959$. Green (red) lines represent the old (new) DFS models. 

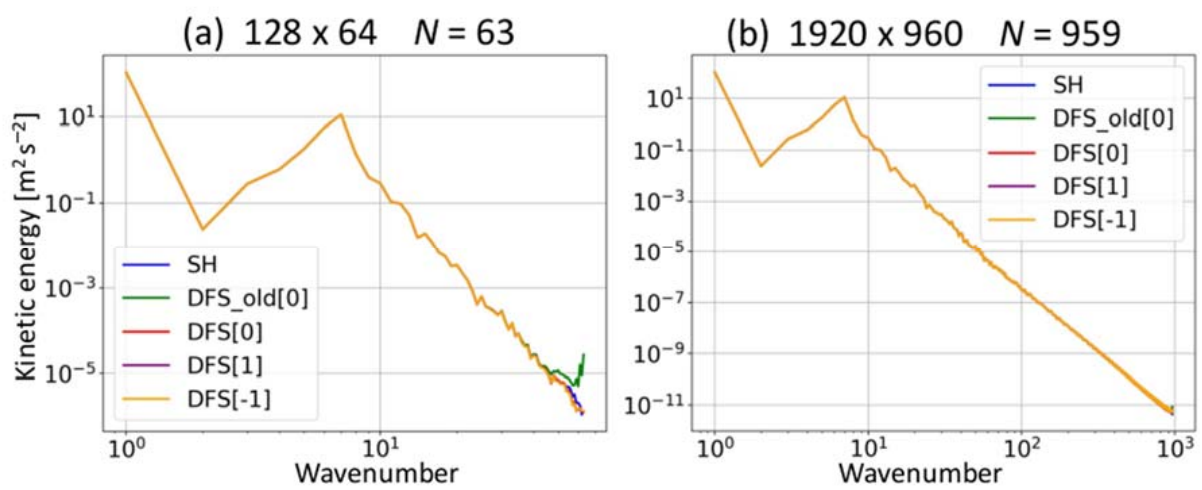

Figure 6. Kinetic energy spectrum of horizontal winds $\left(\mathrm{m}^{2} \mathrm{~s}^{-2}\right)$ after a 15-day integration in Williamson test case 5. Results

5 of the models with (a) around $128 \times 64$ grid points and $N=63$ (DFS) or $N=62$ (SH), and (b) around $1920 \times 960$ grid points and $N=959$ or 958 . The colors blue, green, red, purple, and orange represent the models using SH, old DFS with Grid [0], new DFS with Grid [0], new DFS with Grid [1], and new DFS with Grid [-1], respectively. 
https://doi.org/10.5194/gmd-2021-168

Preprint. Discussion started: 8 July 2021

(c) Author(s) 2021. CC BY 4.0 License.

(c) (1)

(a) DFS [0] $128 \times 64 \mathrm{~N}=42$

(b) DFS [1] $128 \times 65 \quad \mathrm{~N}=42$

(c) DFS [-1] $128 \times 63 \mathrm{~N}=42$
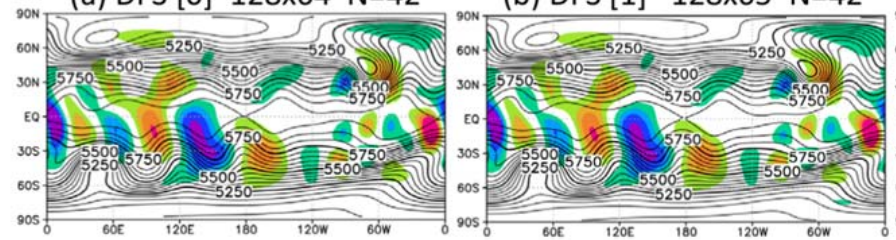

(c) DFS [-1] $128 \times 63 \mathrm{~N}=42$

(d) DFS_old [0] $128 \times 64 \mathrm{~N}=42$

Unstable

(e) $\mathrm{SH} 128 \times 64 \quad \mathrm{~N}=42$
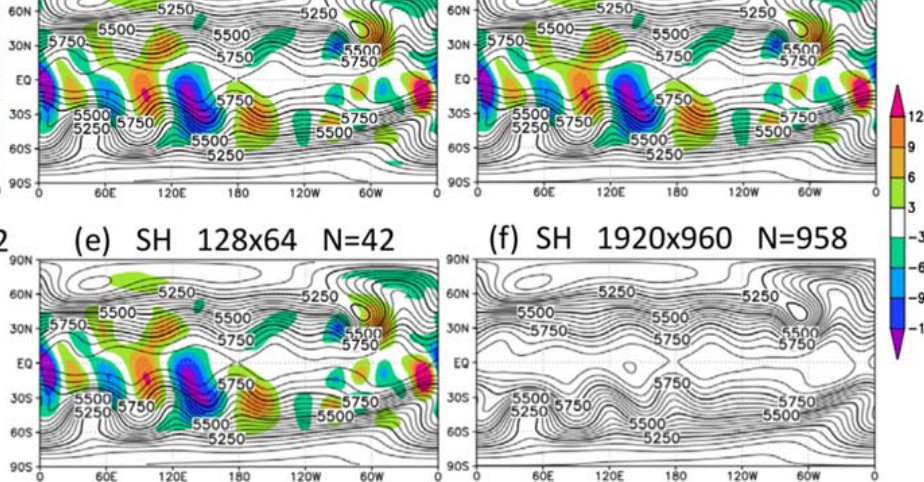

(f) $\mathrm{SH} 1920 \times 960 \mathrm{~N}=958$
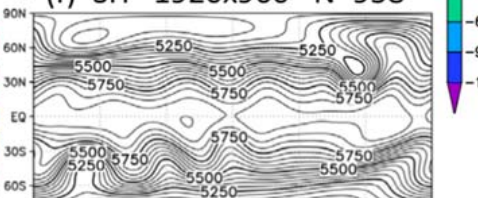

Figure 7. Same as Fig. 4, except with truncation wavenumber $N$. 
https://doi.org/10.5194/gmd-2021-168

Preprint. Discussion started: 8 July 2021

(c) Author(s) 2021. CC BY 4.0 License.

(c) (i)

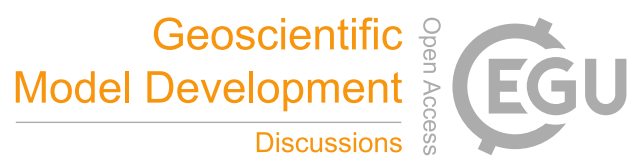

(a) $128 \times 64 \quad N=42$

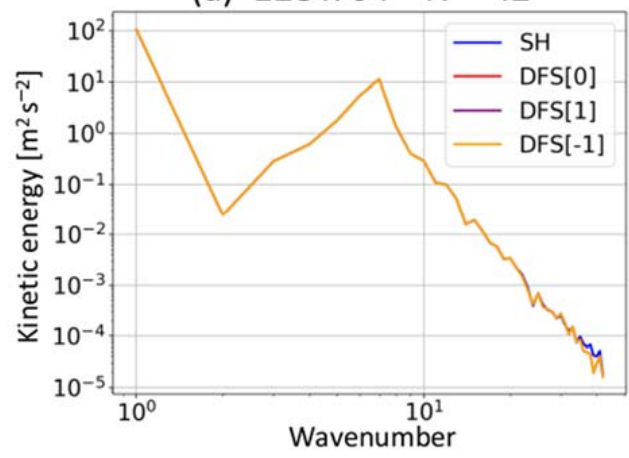

(b) $1920 \times 960 \quad N=639$

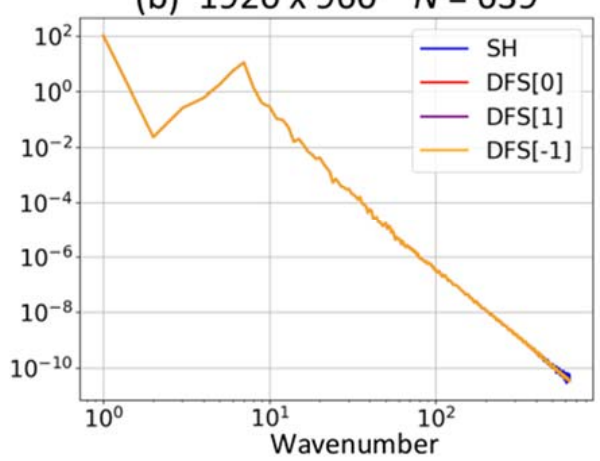

Figure 8. Same as Fig. 6, except with truncation wavenumber $N$. 
(a) DFS [0] $30720 \times 15360 N=10239$

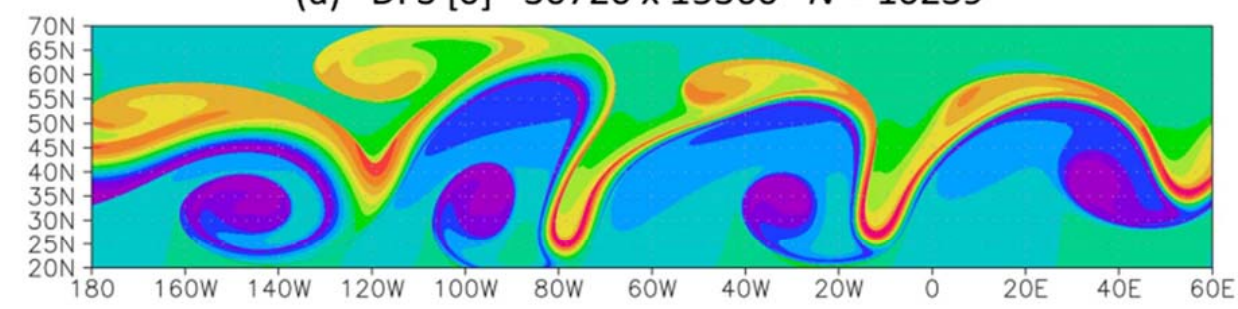

(b) SH $30720 \times 15360 N=10239$

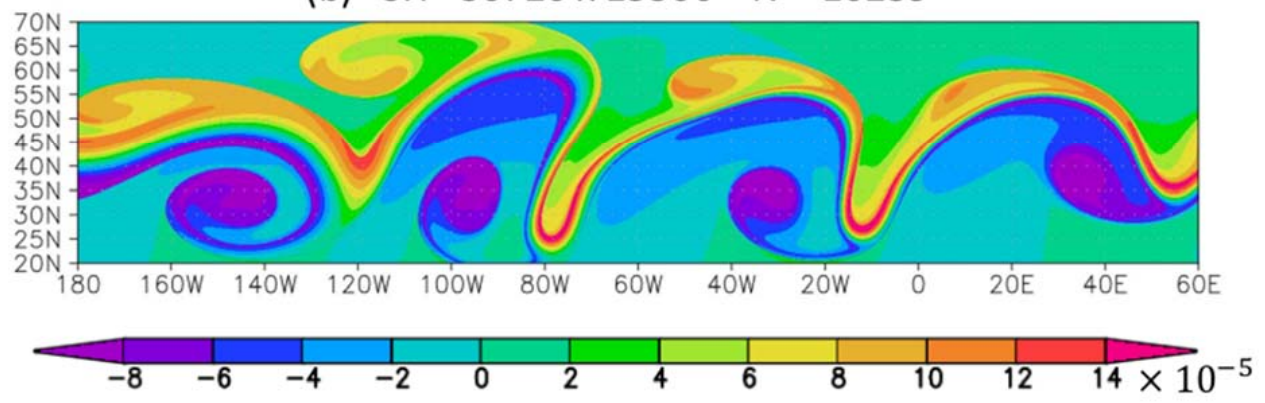

Figure 9. Predicted vorticity $\left(\mathrm{s}^{-1}\right)$ after a 6-day integration in the Galewsky test case. (a) The new DFS model with Grid [0], and (b) the SH model at $1.3 \mathrm{~km}$ resolution with $30720 \times 15360$ grid points and $N=10239$. 
(a) $30720 \times 15360 \quad N=10239$

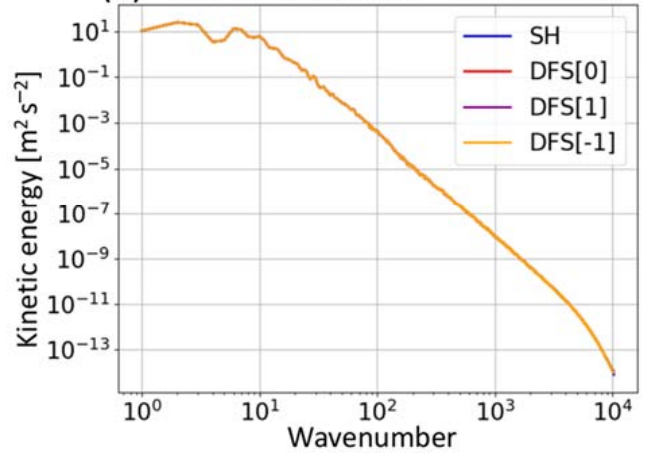

(b) High wavenumber region

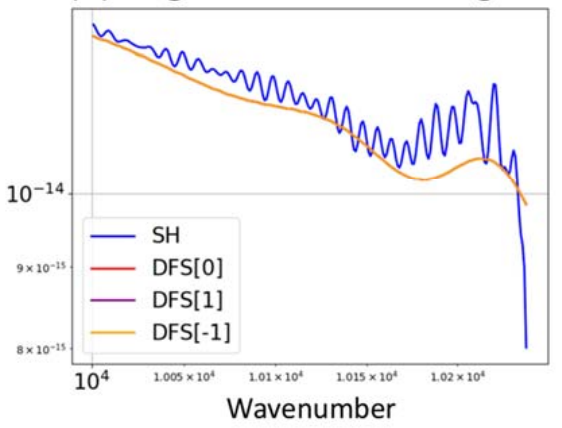

Figure 10. Kinetic energy spectrum of horizontal winds $\left(\mathrm{m}^{2} \mathrm{~s}^{-2}\right)$ after a 6-day integration in the Galewsky test case. (a) Results of the models with $30720 \times 15360$ grid points. The colors blue, green, and red represent the models using SH, old DFS 5 with Grid [0], and DFS with Grid [0], respectively. (b) As (a), but showing the high-wavenumber region. 
https://doi.org/10.5194/gmd-2021-168

Preprint. Discussion started: 8 July 2021

(c) Author(s) 2021. CC BY 4.0 License.

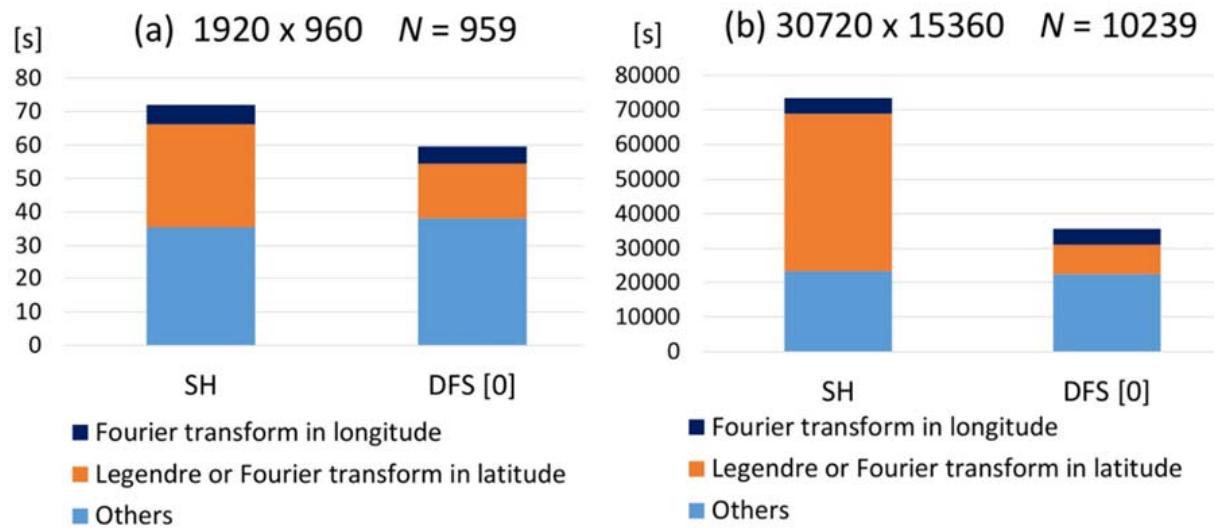

Figure 11. Elapsed time (s) for (a) 15-day integration in Williamson test case 5 in the SH model and the new DFS model at $20 \mathrm{~km}$ resolution with $1920 \times 960$ grid points, and (b) 6-day integration in the Galewsky test case at $1.3 \mathrm{~km}$ resolution with $530720 \times 15360$ grid points. There is no monitoring output during elapsed time measurement. 